\title{
Artificial Three-Body Equilibria for Hybrid Low-Thrust Propulsion
}

\author{
Shahid Baig*and Colin R. McInnes ${ }^{\dagger}$ \\ Department of Mechanical Engineering, University of Strathclyde, Glasgow, G1 1XJ, Scotland, UK.
}

This paper proposes a new concept of creating artificial equilibrium points in the circular restricted three body problem, where the third body uses a hybrid of solar sail and solar electric propulsion. The work aims to investigate the use of a hybrid sail for artificial equilibrium points that are technologically difficult with either of these propulsion systems alone. The hybrid sail has freedom in specifying the sail lightness number, then minimizing the required thrust acceleration from the solar electric propulsion thruster while satisfying the equilibrium condition. The stability analysis of such artificial equilibrium points by a linear method results in a linear time varying (mass) system. The freezing time method then provides unstable and marginally stable regions for hybrid solar sail artificial equilibria. We compare these propulsion systems with a given payload mass and mission life for a polar observation mission. For a near term sail assembly loading we find for the hybrid sail a substantially lower propellant mass compared to solar electric propulsion and lower sail length with respect to a solar sail, and a lower initial spacecraft mass.

\section{Nomenclature}

$A_{S} \quad$ sail area (not including thin film area)

$A_{T F}$ thin film area

$A_{T} \quad$ total hybrid sail area, $=A_{S}+A_{T F}$

$a_{\text {ref }}$ dimensional reference acceleration, $=0.00593 \mathrm{~m} / \mathrm{s}^{2}$ in the Sun-Earth system

$a_{T} \quad$ nondimensional thrust acceleration from electric propulsion system of the hybrid sail

$a_{T} \quad$ dimensional thrust acceleration from electric propulsion system of the hybrid sail, $=a_{\text {ref }} \times a_{T}$

$a_{s} \quad$ nondimensional acceleration magnitude due to solar radiation pressure for a hybrid sail

$\mathbf{a}_{g c}$ nondimensional required acceleration vector to balance gravitational and centrifugal force

${ }^{*}$ PhD Candidate, shahid.baig@strath.ac.uk

${ }^{\dagger}$ Professor, colin.mcinnes@strath.ac.uk, member AIAA 


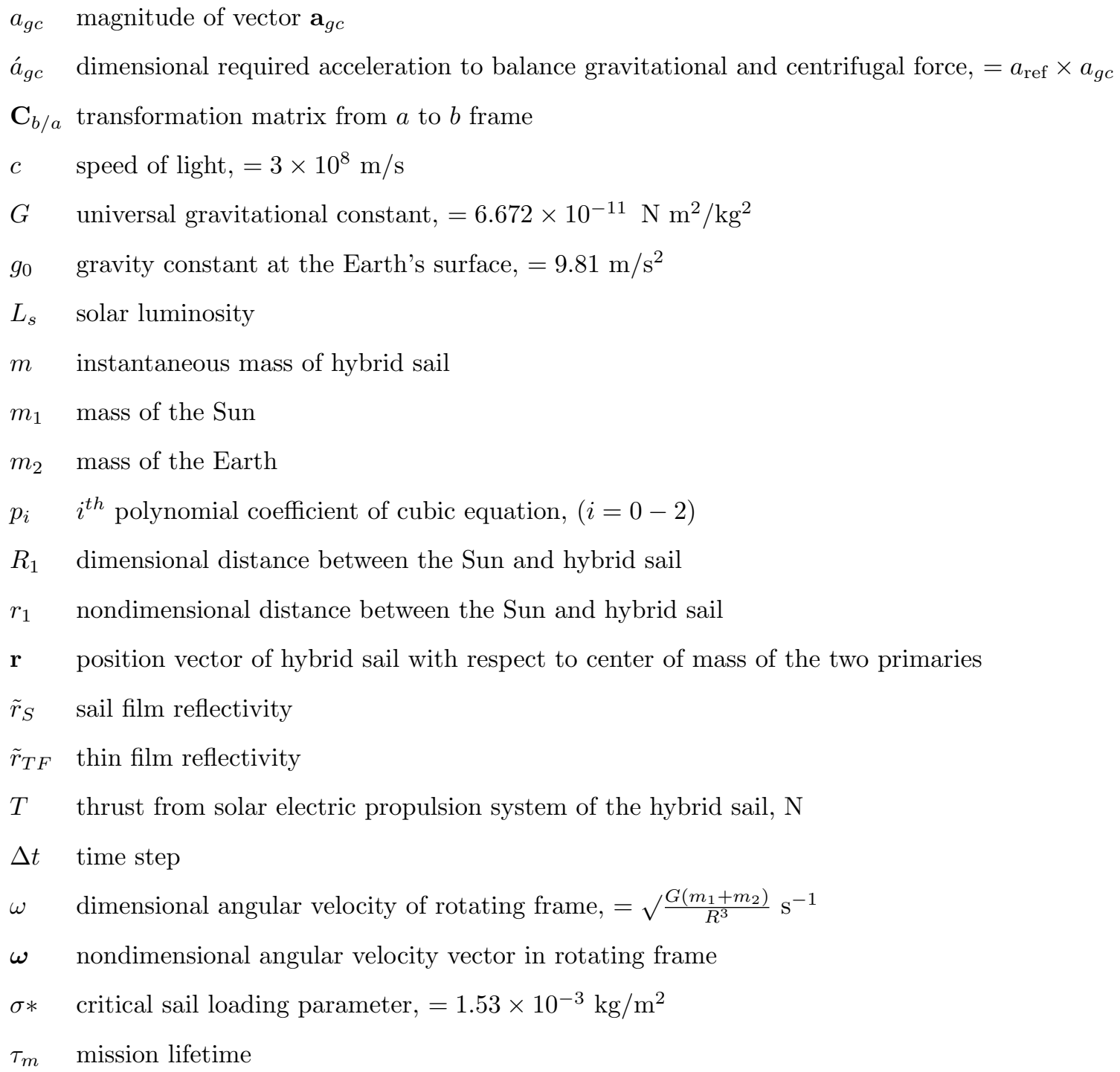

\section{Introduction}

Five natural equilibrium points exist in the classical circular restricted three body problem (CRTBP). These points are the Lagrange points where gravitational and centrifugal forces acting on a spacecraft in a rotating frame are balanced. Artificial equilibrium points (AEPs) similar to Lagrange points can be generated if continuous constant acceleration is available from a low-thrust propulsion system such as solar sail or solar electric propulsion (SEP). The continuous acceleration from either of these propulsion systems cancels any residual acceleration at the AEP, so that a static equilibrium point can be generated or a periodic orbit around the AEP if the eigenvalue spectrum of the AEP contains at least one centre. For a solar sail, incident and reflected solar photons transfer momentum to a large and lightweight reflective membrane and so add a low thrust continuous acceleration in the CRTBP. McInnes et al. ${ }^{1}$ show that continuous surfaces of 
unstable AEPs are generated. These AEPs are characterized by sail lightness number (or total sail loading) and sail orientation. McInnes ${ }^{2}$ in subsequent studies finds for a realistic, partially reflecting sail where the acceleration vector is no longer strictly normal to the sail surface, a reduction in the volume of artificial equilibrium solutions attached to the natural $L_{1}$ and $L_{2}$ Lagrange points. Morrow et al. ${ }^{3}$ carried out analysis for a solar sail hovering in close proximity to an asteroid and found AEP solutions in Hill's problem similar to the restricted three-body problem. Baoyin and McInnes ${ }^{4}$ reconsider AEP solutions for a solar sail in the elliptical restricted three-body problem and find equilibrium points exist only within the ecliptic plane. However, the authors find that when the eccentricity of the orbit of the primary bodies is small, out of ecliptic equilibria can be achieved with active control. Other studies ${ }^{5,6}$ have suggested periodic orbits around these AEPs in and above the ecliptic plane for the solar sail CRTBP. The NASA/NOAA Geostorm warning mission is an application of solar sail equilibria in the ecliptic plane and is based on a sail assembly loading of $14 \mathrm{~g} / \mathrm{m}^{2}$ (a key sail technology parameter) and sail size of $100 \times 100 \mathrm{~m}^{7}$ NOAA interest $^{8}$ in polar observer missions ${ }^{9,10}$ uses an application of sail equilibria out of the ecliptic plane. However, for a polar observer mission, a sail with the same sail assembly loading as for the Geostorm mission needs a large sail $(>175 \times 175 \mathrm{~m})$. The deployment and control of such a large solar sail will be technologically difficult. Improvements in two key sail design parameters, a decrease in the sail assembly loading and an increase in sail length are being developed. ${ }^{11}$

For an SEP system, where reaction mass provides a low thrust propulsive force, Morimoto et al. ${ }^{12}$ find artificial equilibrium points (unstable and marginally stable) in the CRTBP. These AEPs are characterised by the low thrust acceleration magnitude and thrust orientation. Morimoto et al. ${ }^{13}$ also find resonant periodic orbits at linear order around the marginally stable points along the axis joining the primary bodies with a constant, continuous acceleration. However, continuous acceleration from an SEP system at AEPs for several years will require a large mass of propellant fraction, unlike a sail which is a true propellantless system.

In this paper, we investigate a new concept for creating AEPs in the CRTBP using these two low thrust propulsion systems (solar sail and SEP) simultaneously. Such a hybrid of sail/SEP spacecraft is termed a hybrid sail. We find that a hybrid sail can be in equilibrium in forbidden regions for a pure sail. In particular we consider a polar observer mission and compare the hybrid sail, pure SEP and pure sail systems with a given payload mass and mission life time. We find that for a hybrid sail with the same assembly loading as for the Geostorm mission, substantially lower sail length with respect to a pure sail system and lower propellant mass and maximum electric power with respect to a pure SEP system.

The idea of the hybrid sail was apparently first proposed by Leipold and Götz, ${ }^{14}$ who assume a square sail and an SEP thruster attached to the sail centre, with part of the sail area at the sail center covered by flexible thin film solar cells (TFSC). TFSC will act as a power source for the SEP system and other subsystems of the hybrid sail. TFSC technology has many advantages over state-of-the-art wafer based 
solar cell technology including high power/ mass ratio, low costs and good resistance against radiation. To qualify TFSC for future space use, flight experiments are being planned in Low Earth orbit and Medium Earth orbit. ${ }^{15}$ Leipold and Götz ${ }^{14}$ and recently Mengali and Quarta ${ }^{16}$ in their studies show that the hybrid sail has an attractive feature of reducing mission time with respect to a pure sail and a pure SEP system respectively for heliocentric transfers.

In the next section we describe the total force model for a partially reflecting hybrid sail. The solar radiation pressure (SRP) and SEP accelerations are normalized with respect to a reference acceleration and are used in Sec. III to describe the equations of motion of a hybrid sail in the CRTBP. For a hybrid sail, we find freedom in specifying the sail lightness number at a given AEP and minimizing the required SEP acceleration while satisfying the equilibrium condition. Two strategies are discussed to maintain the equilibrium condition. In Sec. IV, because of the slowly time varying (mass) linear system, the freezing time method is used to determine the stability of AEPs of a hybrid sail in the Sun-Earth CRTBP. Section V compares the performance of the hybrid sail relative to a pure sail and a pure SEP system for a polar Earth observer mission. Finally, conclusions are presented in Sec. VI.

\section{Partially Reflecting Hybrid Sail Model}

\section{A. Dimensional Force Model}

The hybrid sail configuration is adopted from Leipold and Götz ${ }^{14}$ as described in the previous section. They developed a hybrid sail force model that takes different reflectivities for the sail and TFSC area for their magnitude, but leaves the thrust direction acting normal to the sail surface. This paper considers a hybrid sail model which has a SRP force component along the sail surface (non-ideal reflectivity) and so the total SRP force is no longer normal to sail surface.

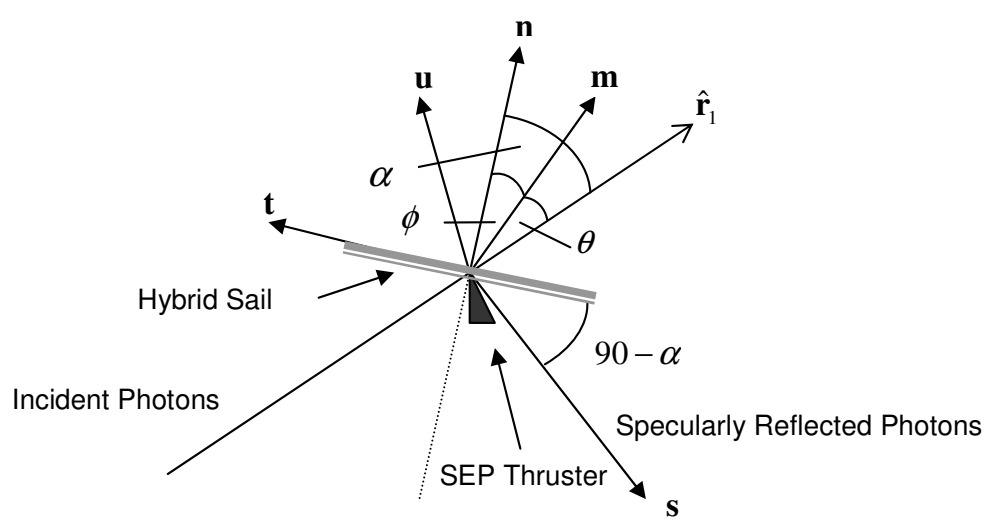

Figure 1. Solar radiation pressure force model for a specularly reflecting hybrid sail. The solar electric propulsion thruster is also shown. 
The solar radiation pressure at a distance $R_{1}$ from the Sun is given by

$$
P=\frac{L_{s}}{4 \pi R_{1}{ }^{2} c}
$$

The unit vectors normal to and transverse to the hybrid sail surface are defined by $\mathbf{n}$ and $\mathbf{t}$ respectively, as shown in Figure 1. The direction of incident photons is described by $\hat{\mathbf{r}}_{1}=\cos \alpha \mathbf{n}-\sin \alpha \mathbf{t}$ and so the SRP force due to the incident photons is then

$$
\mathbf{F}_{i}=P A_{T} \cos \alpha\left(\hat{\mathbf{r}}_{1}\right)
$$

where $A_{T} \cos \alpha$ is the projected area in the direction of the incident photons. We assume specular reflection (no diffuse reflection and thermal re-emission) from both TFSC area $A_{T F}$ and sail area $A_{S}$. The force on the hybrid sail due to the reflected photons is then

$$
\mathbf{F}_{r}=\tilde{r}_{S} P A_{S} \cos \alpha(-\mathbf{s})+\tilde{r}_{T F} P A_{T F} \cos \alpha(-\mathbf{s})
$$

The unit vector $\mathbf{s}=-\cos \alpha \mathbf{n}-\sin \alpha \mathbf{t}$ defines the direction of the specularly reflected photons. The total force exerted on the hybrid sail due to incident and reflected photons is therefore

$$
\mathbf{F}_{S}=F_{n} \mathbf{n}+F_{t} \mathbf{t}
$$

with

$$
\begin{aligned}
F_{n} & =\left(\hat{\mathbf{r}}_{1} \cdot \mathbf{n}\right)^{2}\left[\left(1+\tilde{r}_{S}\right) P A_{S}+\left(1+\tilde{r}_{T F}\right) P A_{T F}\right] \\
F_{t} & =\left(\hat{\mathbf{r}}_{1} \cdot \mathbf{n}\right)\left(\hat{\mathbf{r}}_{1} \cdot \mathbf{t}\right)\left[\left(1-\tilde{r}_{S}\right) P A_{S}+\left(1-\tilde{r}_{T F}\right) P A_{T F}\right]
\end{aligned}
$$

where $\hat{\mathbf{r}}_{1} \cdot \mathbf{n}=\cos \alpha$ and $\hat{\mathbf{r}}_{1} \cdot \mathbf{t}=-\sin \alpha$, and so the SRP force on the hybrid sail $\mathbf{F}_{S}$ will now act in direction $\mathbf{m}$ as shown in Figure 1.

The force due to the SEP thruster placed at the centre of the sail, as shown in Figure 1, is given by

$$
\mathbf{F}_{S E P}=T \mathbf{u}
$$

where the unit vector $\mathbf{u}$ denotes the thrust direction.

The total thrust provided by the hybrid sail due to the SRP and the SEP thruster can be obtained from the sum of Eqs. (4) and (5).

\section{B. Nondimensional Acceleration Model}

In the CRTBP, a spacecraft of negligible mass $m$ moves under the gravitational influence of the two primaries. The two primaries $m_{1}$ and $m_{2}$ orbit circularly with constant angular velocity $\omega$ in the (ecliptic) plane about their common center of mass. The unit of length is chosen such that the distance between the primaries $R$ is

5 of 27 
taken to be unity and the unit of mass is chosen such that $G\left(m_{1}+m_{2}\right)=1$. If we define $\mu=\frac{m_{2}}{m_{1}+m_{2}}$ as a nondimensional mass ratio, then in this system the gravitational constants are $G m_{2}=\mu$ and $G m_{1}=1-\mu$. The orbit period of the primary bodies is set to $\tau=2 \pi$. Thus the nondimensional unit acceleration corresponds to $a_{\text {ref }}=\omega^{2} R=0.00593 \mathrm{~m} / \mathrm{s}^{2}$ in the Sun-Earth system.

To obtain the acceleration $\mathbf{a}_{S}$ due to SRP for a hybrid sail in non-dimensional form, which will be used in equations of motion of the hybrid sail described in the next section, we divide Eq. (4) by mass $m$ and dimensional reference acceleration $\omega^{2} R$, then re-arranging we have

$$
\mathbf{a}_{S}=a_{s} \mathbf{m}=\frac{1}{2} \beta_{0} \frac{m_{0}}{m} \frac{1-\mu}{r_{1}^{2}} g\left(\hat{\mathbf{r}}_{1} \cdot \mathbf{n}\right)^{2} \mathbf{n}+\frac{1}{2} \beta_{0} \frac{m_{0}}{m} \frac{1-\mu}{r_{1}^{2}} h\left(\hat{\mathbf{r}}_{1} \cdot \mathbf{n}\right)\left(\hat{\mathbf{r}}_{1} \cdot \mathbf{t}\right) \mathbf{t}
$$

where

$$
\begin{aligned}
& g=\left(1+\tilde{r}_{S}\right)-\frac{A_{T F}}{A_{T}}\left(\tilde{r}_{S}-\tilde{r}_{T F}\right) \\
& h=\left(1-\tilde{r}_{S}\right)+\frac{A_{T F}}{A_{T}}\left(\tilde{r}_{S}-\tilde{r}_{T F}\right)
\end{aligned}
$$

and $m_{0}$ is the initial mass of hybrid sail and $\beta_{0} \equiv \frac{\sigma^{*}}{\left(\frac{m_{0}}{A_{T}}\right)}$ is defined as the dimensionless lightness number. The acceleration model for a non-ideal pure sail ${ }^{2}$ is easily recovered from Eq. (6) when the sail mass is constant $m=m_{0}$ and $\tilde{r}_{T F}=\tilde{r}_{S}$.

For a given $m_{0}, \beta_{0}$ and TFSC fractional area with respect to total area, the magnitude of acceleration due to SRP acting on the hybrid sail increases with the decrease of hybrid sail mass $m$ and may be written as

$$
a_{s}=\frac{1}{2} \beta_{0} \frac{m_{0}}{m} \frac{1-\mu}{r_{1}^{2}} \sqrt{g^{2} \cos ^{2} \alpha+h^{2} \sin ^{2} \alpha} \cos \alpha
$$

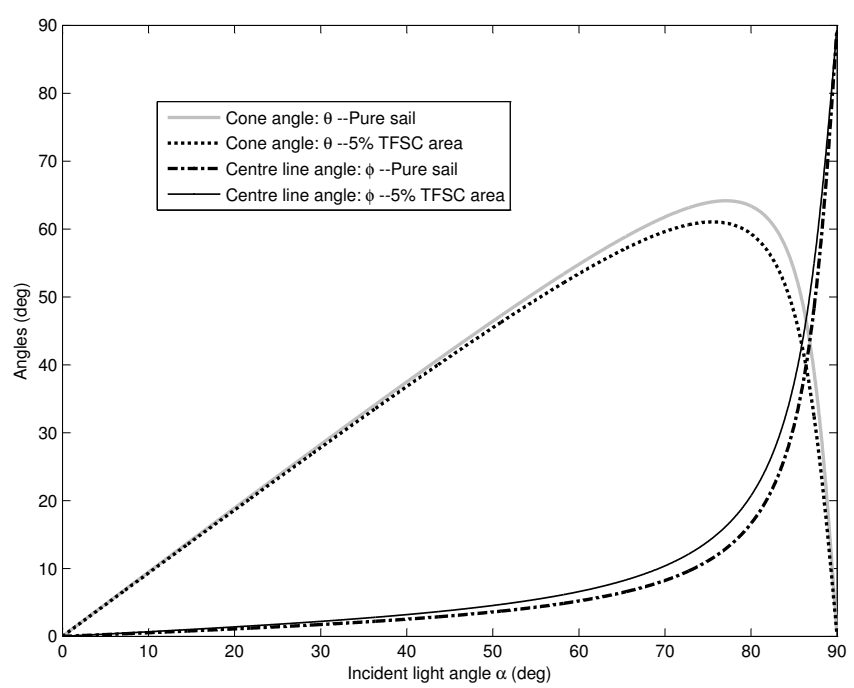

Figure 2. Maximum cone angle for a hybrid sail (5\% TFSC area w.r.t total area) is less than that for a pure sail (with no TFSC area)

6 of 27 
The offset angle between $\mathbf{m}$ and $\mathbf{n}$ usually called centre line angle $\phi$ (see Figure 1) can be obtained from Eq. (6) by dividing the ratio of transverse and normal accelerations as

$$
\tan \phi=\frac{h}{g} \tan \alpha
$$

The actual direction of the SRP acceleration for a hybrid sail is defined by the cone angle $\theta$. Using the relation $\alpha=\theta+\phi$ and Eq. (8), the cone angle $\theta$ can be written as

$$
\tan \theta=\frac{(g-h) \tan \alpha}{g+h \tan ^{2} \alpha}
$$

We assume a reflectivity for a typical aluminized sail film $\tilde{r}_{S}=0.9$ and for the TFSC area $\tilde{r}_{T F}=0.4 .^{14}$ Figure 2 shows that the maximum cone angle $\theta_{\max }=61 \mathrm{deg}$ of the hybrid sail is less than the maximum cone angle $64.15 \mathrm{deg}$ of a pure sail. This is due to the fact that for a hybrid sail $\tilde{r}_{T F}<\tilde{r}_{S}$.

The non-dimensional acceleration due to the SEP thruster can be obtained from Eq. (5) by dividing through by the hybrid sail mass $m$ and reference acceleration $a_{\text {ref }}$ as

$$
\mathbf{a}_{S E P}=\frac{T / m}{a_{\mathrm{ref}}} \mathbf{u}=a_{T} \mathbf{u}
$$

These force models will now be used to define the sets of AEP.

\section{Equations of Motion and Artificial Equilibria}

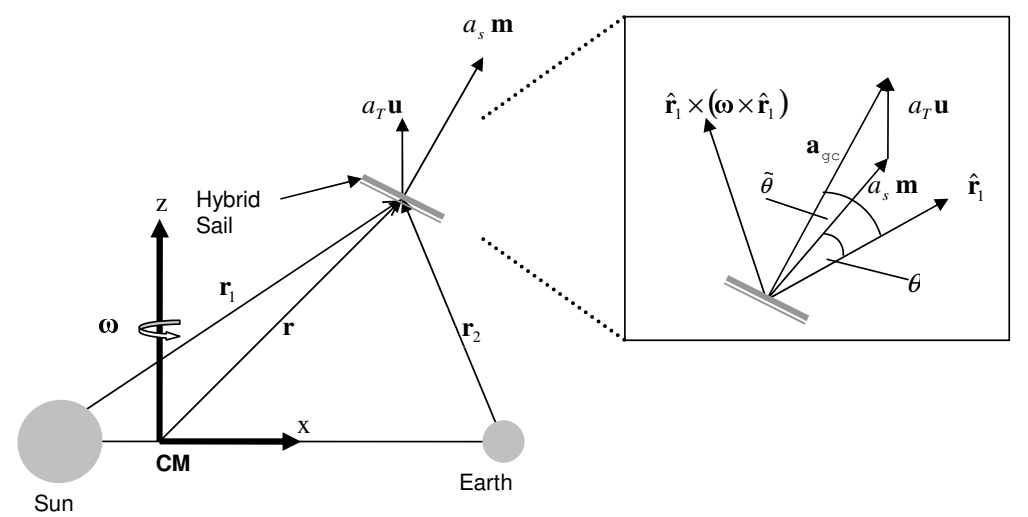

Figure 3. Definition of coordinate system and hybrid sail using two low-thrust propulsion systems.

Consider a frame $F_{a}(x, y, z)$ co-rotating with the two primary masses at constant angular velocity $\boldsymbol{\omega}$ with origin at their center of mass, as shown in Figure 3. The x-axis points along the Sun-Earth line, the z-axis is the axis of rotation and the $\mathrm{y}$-axis completes the right-handed coordinate system. The nondimensional equation of motion of a hybrid sail in the rotating frame of reference $F_{a}$ is given by

$$
\begin{gathered}
\frac{d^{2} \mathbf{r}}{d t^{2}}+2 \boldsymbol{\omega} \times \frac{d \mathbf{r}}{d t}+\boldsymbol{\omega} \times(\boldsymbol{\omega} \times \mathbf{r})=-\nabla V+\mathbf{a}_{S}+\mathbf{a}_{S E P} \\
7 \text { of } 27
\end{gathered}
$$


where $\boldsymbol{\omega}=\left[\begin{array}{lll}0 & 0 & 1\end{array}\right]^{T}$. The vectors $\mathbf{a}_{S}$ and $\mathbf{a}_{S E P}$ are the accelerations due to solar radiation pressure and the solar electric propulsion system and may be written in the frame $F_{a}$ as

$$
\mathbf{a}_{S}=a_{s} \mathbf{m}^{a}, \quad \mathbf{a}_{S E P}=a_{T} \mathbf{u}^{a}
$$

The three-body gravitational potential $V$ is defined as

$$
V=-\left[\frac{1-\mu}{r_{1}}+\frac{\mu}{r_{2}}\right]
$$

where $\mathbf{r}_{1}=\left[\begin{array}{lll}x+\mu & y & z\end{array}\right]^{T}$ and $\mathbf{r}_{2}=\left[\begin{array}{lll}x-(1-\mu) & y & z\end{array}\right]^{T}$ are the position vectors of the hybrid sail with respect to the primary bodies. The centrifugal term in Eq. (11) can be written as

$$
\nabla \Phi(\mathbf{r})=\boldsymbol{\omega} \times(\boldsymbol{\omega} \times \mathbf{r}), \quad \Phi(\mathbf{r})=-\frac{1}{2}\left(x^{2}+y^{2}\right)
$$

By defining a new scalar function $U(\mathbf{r})=V(\mathbf{r})+\Phi(\mathbf{r})$, the reduced equation of motion for the hybrid sail is obtained as

$$
\frac{d^{2} \mathbf{r}}{d t^{2}}+2 \boldsymbol{\omega} \times \frac{d \mathbf{r}}{d t}+\nabla U(\mathbf{r})=\mathbf{a}_{S}+\mathbf{a}_{S E P}
$$

An artificial equilibrium point $\mathbf{r}_{0}$ in the rotating frame of reference $F_{a}$ is obtained if the vector sum of the continuous low thrust acceleration from the two propulsion systems satisfying the following equation

$$
\nabla U\left(\mathbf{r}_{0}\right)=\mathbf{a}_{S}+\mathbf{a}_{S E P} \triangleq \mathbf{a}_{g c}
$$

Now $\nabla U\left(\mathbf{r}_{0}\right)=\mathbf{a}_{g c}$ is the required acceleration vector to cancel the gravitational forces of the two primary bodies and the centrifugal force in the rotating frame $F_{a}$. It may also be defined as the required acceleration for converting a nonequilibrium point into an AEP at $\mathbf{r}_{0}$. For a pure sail system, ${ }^{1}$ the required acceleration vector is generated by the SRP acceleration vector alone, while for a pure SEP system ${ }^{12}$ it is generated by the acceleration vector from the SEP system alone. For a hybrid sail, Eq. (16) shows that the required vector to keep the hybrid sail at AEP $\mathbf{r}_{0}$ is generated by the vector sum of the SRP and the SEP acceleration vectors. We now define a new frame $F_{b}$, that will be useful in the next section to minimize the thrust acceleration from the SEP system. $F_{b}$ is defined with a set of three orthogonal vectors $\left\{\mathbf{r}_{1}, \boldsymbol{\omega} \times \mathbf{r}_{1}, \mathbf{r}_{1} \times\left(\boldsymbol{\omega} \times \mathbf{r}_{1}\right)\right\}$ and with its origin at the hybrid sail position. The rotation matrix from $a$ to $b$ can then be written as

$$
\mathbf{C}_{b / a}\left(\mathbf{r}_{0}\right)=\left[\begin{array}{lll}
\frac{\mathbf{r}_{1}}{\left|\mathbf{r}_{1}\right|} & \frac{\boldsymbol{\omega} \times \mathbf{r}_{1}}{\left|\boldsymbol{\omega} \times \mathbf{r}_{1}\right|} & \frac{\mathbf{r}_{1} \times\left(\boldsymbol{\omega} \times \mathbf{r}_{1}\right)}{\left|\mathbf{r}_{1} \times\left(\boldsymbol{\omega} \times \mathbf{r}_{1}\right)\right|}
\end{array}\right]^{T}
$$

Therefore, the condition for artificial equilibrium Eq. (16), in $F_{b}$ is given by

$$
\mathbf{a}_{g c}^{b}=a_{s} \mathbf{m}^{b}+a_{T} \mathbf{u}^{b}
$$


where $\mathbf{a}_{g c}^{b}=\left[\begin{array}{lll}a_{1} & a_{2} & a_{3}\end{array}\right]^{T}=\mathbf{C}_{b / a} \nabla U\left(\mathbf{r}_{\mathbf{0}}\right)$. Eq. (18) can now be rewritten as

$$
a_{T}^{2}=a_{g c}^{2}-2 a_{s} \mathbf{m}^{b} \cdot \mathbf{a}_{g c}^{b}+a_{s}^{2}
$$

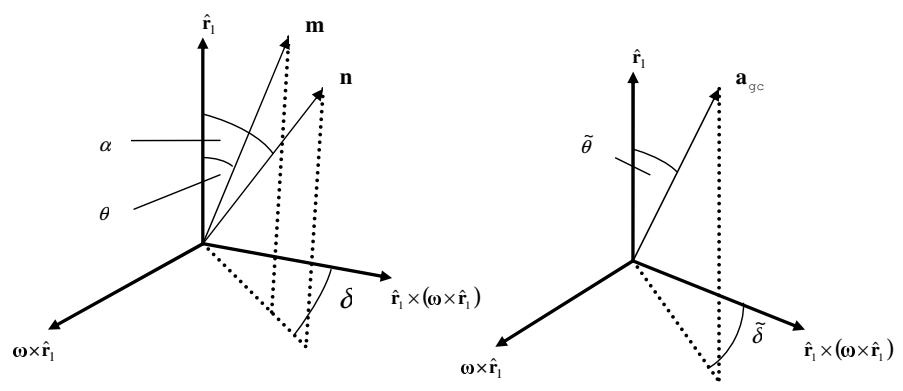

Figure 4. Definition of cone and clock angles for vectors $\mathbf{n}, \mathbf{m}$ and $\mathbf{a}_{g c}$ in frame $F_{b}$

The required vector $\mathbf{a}_{g c}$ to keep the hybrid sail at an AEP, and the direction $\mathbf{m}$ that defines the direction of the SRP acceleration vector can be expressed in $F_{b}$ according to Fig. 4 as

$$
\mathbf{a}_{g c}^{b}=a_{g c}\left[\begin{array}{r}
\cos \widetilde{\theta} \\
\sin \widetilde{\theta} \sin \widetilde{\delta} \\
\sin \widetilde{\theta} \cos \widetilde{\delta}
\end{array}\right], \quad \mathbf{m}^{b}=\left[\begin{array}{r}
\cos \theta \\
\sin \theta \sin \delta \\
\sin \theta \cos \delta
\end{array}\right]
$$

where the cone angle $\widetilde{\theta}$ and clock angle $\widetilde{\delta}$ of $\mathbf{a}_{g c}$ depend upon the AEP $\mathbf{r}_{0}$ and can be calculated as

$$
\begin{aligned}
\widetilde{\theta} & =\cos ^{-1}\left(\frac{a_{1}}{a_{g c}}\right) \\
\widetilde{\delta} & =\tan ^{-1}\left(a_{2}, a_{3}\right)
\end{aligned}
$$

Using Eq. (20) in Eq. (19), the low thrust acceleration from the SEP system of the hybrid sail can be expressed in terms of the sail pitch angle $\alpha$ and the sail clock angle $\delta$ that defines the hybrid sail normal $\mathbf{n}$ as shown in Fig. 4 as

$$
a_{T}^{2}(\alpha, \delta)=a_{g c}^{2}-2 a_{g c} a_{s}(\cos \theta \cos \widetilde{\theta}+\sin \theta \sin \widetilde{\theta} \cos (\delta-\widetilde{\delta}))+a_{s}^{2}
$$

where $a_{s}$ and $\theta$ are functions of the pitch angle $\alpha$ as given by Eqs.(7) and (9) respectively.

For a pure sail or a pure SEP system the required acceleration magnitude and thrust orientation are completely defined by the location of the artificial equilibria $\mathbf{r}_{0}$. For a hybrid sail, the desired acceleration vector to keep the hybrid sail at an AEP is achieved by the sum of the SRP acceleration vector and the SEP acceleration vector, as shown in Fig. 3. By fixing $\beta_{0}, m=m_{0}$, Fig. 5 shows that there is freedom in selecting the orientation of the SRP acceleration direction to obtain the desired acceleration vector $\mathbf{a}_{g c}$ whilst minimizing the SEP thrust. Once the optimum orientation is selected to obtain the maximum benefit 


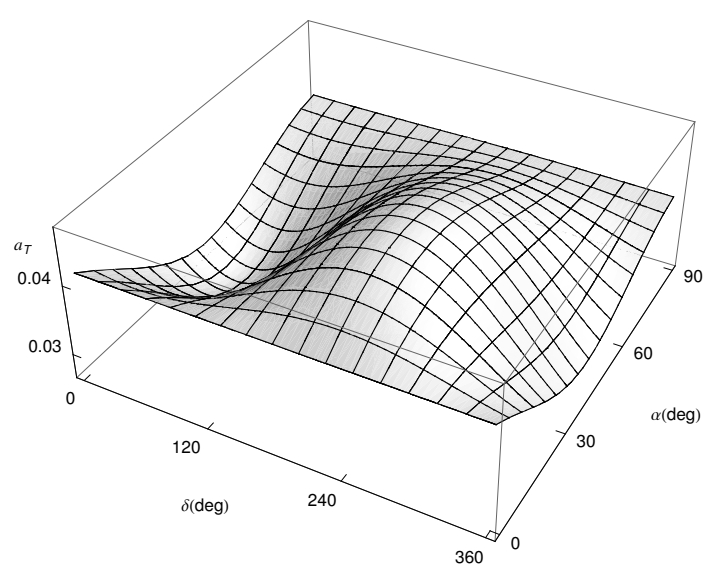

Figure 5. Required low thrust acceleration from SEP at $\mathbf{r}_{0}=\left[\begin{array}{llll}1.005 & 0.005 & 0.005\end{array}\right]^{T}$ as a fuction of the sail pitch angle $\alpha$ and sail clock angle $\delta$ for a sail with $\beta_{0}=0.03$. Minimum $a_{T}=0.0269$ at optimal angles $\left(\alpha^{*}(0), \delta^{*}(0)\right)=$ $\left(40.23^{\circ}, 39.46^{\circ}\right)$.

from the SRP, the required orientation for the SEP thruster system $\mathbf{u}$ may be determined from condition for artificial equilibria Eq. (18).

\section{A. Minimization of SEP Acceleration at $t=0$}

The problem now may be formulated to determine the optimal hybrid sail cone and clock angles $\left(\alpha^{*}(0), \delta^{*}(0)\right)$ to minimize the thrust acceleration $a_{T}$ from the SEP system at an AEP $\mathbf{r}_{0}$ and for a given sail lightness number $\beta_{0}$. At initial time $\mathrm{t}=0, m=m_{0}$ and Eq. (7) becomes

$$
a_{s}=a_{s}(0)=\frac{1}{2} \beta_{0} \frac{1-\mu}{r_{1}^{2}} \sqrt{g^{2} \cos ^{2} \alpha+h^{2} \sin ^{2} \alpha} \cos \alpha
$$

Using Eq. (24) in Eq. (23) and setting the derivative of $a_{T}$ with respect to $\delta$ to zero yields a stationary point for the optimal clock angle as

$$
\frac{\partial a_{T}}{\partial \delta}=a_{g c} \frac{a_{s}(0)}{a_{T}} \sin \theta \sin \widetilde{\theta} \sin (\delta-\widetilde{\delta})=0
$$

With $\alpha \neq 90 \operatorname{deg}$ or $a_{s}(0) \neq 0, \theta \neq 0$ and $\widetilde{\theta} \neq 0$, Eq. (25) holds if

$$
\delta^{*}(0)=\widetilde{\delta}
$$

This states that the hybrid sail clock angle should be aligned with the clock angle of the vector $\mathbf{a}_{g c}$ in order to minimize the thrust acceleration from the SEP system. Inserting this result into Eq. (23) yields

$$
a_{T}^{2}(\alpha)=a_{g c}^{2}-2 a_{g c} a_{s}(0) \cos (\widetilde{\theta}-\theta)+a_{s}^{2}(0)
$$

The above equation can be minimized numerically for $\alpha^{*}(0)$, for example by using Mathematica ${ }^{\circledR}$ and specifying the bounds for $\alpha \in[0, \pi / 2]$. 

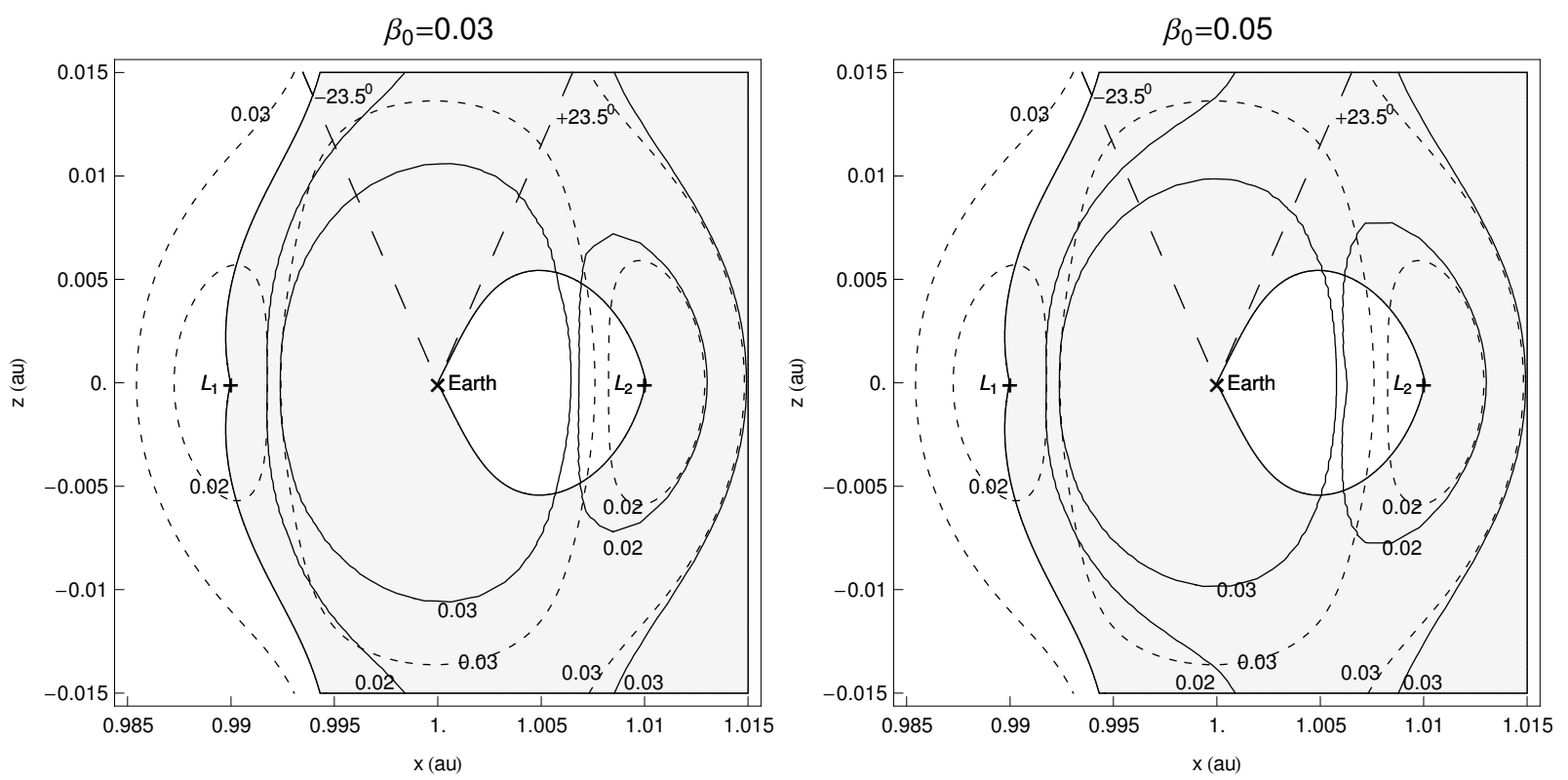

Figure 6. Thrust acceleration contours $a_{T}$ in the xz-plane. Values $0.02,0.03$ shown by dotted lines for pure SEP system and shown by solid lines for the hybrid sail. $\mp 23.5 \mathrm{deg}$ are angles of polar axis of Earth (dashed lines) with respect to the normal to the ecliptic plane at summer and winter solstices.

To show the feasibility of the hybrid sail for some practical missions to be discussed in Sec. V, we will now only consider the analysis in the xz-plane $\left(\delta^{*}=\widetilde{\delta}=0\right.$ if $a_{3}>0$, or $\delta^{*}=\widetilde{\delta}=\pi$ if $\left.a_{3}<0\right)$. Fig. 6 shows families of thrust acceleration contours near the Earth in the case of pure SEP and a hybrid sail. The shaded area shows the region where the pure sail cannot be placed as the SRP force direction is constrained by the maximum cone angle $\left(\theta \leq \theta_{\max }\right)$ due to the sail film partial reflectivity. The benefit of the hybrid sail is clear over the pure sail. The pure sail can be placed in the shaded region by combining it with an electric thruster, since the thrust vector from the SEP system of a hybrid sail can be oriented in any direction. Also, the benefit of the hybrid sail is clear over pure SEP, since a larger volume of space is available for artificial equilibrium solutions around $L_{2}$ and displaced equilibrium solutions towards Earth near $L_{1}$ exist, with the same low thrust acceleration value $\left(a_{T}=0.02\right)$. This is due to part of the total acceleration, to cancel the gravitational forces of two primary bodies and centrifugal force, being available from the solar sail. In general, the addition of a small SEP system to a solar sail allows the hybrid sail to be in equilibrium closer the Earth, and in volumes of space inaccessible to a pure sail system.

In Fig. 6 we can compare the electric thruster acceleration contours in the case of the pure SEP and the hybrid sail system. The electric thruster acceleration contour of value $a_{T}=0.03$ about the Earth in the case of the hybrid system is not symmetric. This depends upon where the hybrid sail can or cannot use SRP effectively. However, the electric thruster acceleration contours of values $|\nabla U|=0.03$ around the Earth for pure SEP are symmetric due to the near symmetric 3-body potential at the Earth. From Earth towards $L_{1}$ along the x-axis, and also in regions beyond $L_{2}$, the acceleration contours of the hybrid sail and the pure 
SEP are identical because at these locations the hybrid sail cannot use SRP effectively. Here the sail pitch angle becomes approximately $90 \mathrm{deg}$ to minimize the required thrust acceleration from the SEP system. At these locations the hybrid sail will not be of use as compared to a pure SEP system.

\section{B. Equilibria Options During Mission Life}

The hybrid sail is a variable mass system, unlike a pure sail which is a constant mass system. As the SEP system consumes propellant, so the magnitude of the SRP acceleration continuously increases with time due to the decrease of the hybrid sail mass $m$. In principle, the thrust magnitude needed from the SEP system should decrease with mission lifetime. There are two options to keep the hybrid sail at an AEP $\mathbf{r}_{0}$ during its mission life.

\section{Option 1}

In option $1, a_{T}$ can be minimized at $t=0$ or for initial mass $m=m_{0}$ as in the previous section, and the hybrid sail can be maintained at this optimum fixed attitude $\left(\alpha^{*}(0), \delta^{*}(0)\right)$ during the whole mission lifetime. Due to the increase of the SRP acceleration $a_{s}$, the thrust from the SEP system should be adjusted in magnitude (throttled) and its direction trimmed at each instant to ensure that the equilibrium condition is satisfied. The algorithm works as follows:

(1) At $t=0, m=m_{0}$, choose appropriate $\beta_{0}$ so that the total sail area $A_{T}=\frac{m_{0} \beta_{0}}{\sigma^{*}}$

(2) In the xz-plane choose an AEP $\mathbf{r}_{0}$ which in turn determines the desired acceleration vector $\mathbf{a}_{g c}$. Calculate the cone angle $\widetilde{\theta}$ using Eq. (21). However, in the xz-plane $a_{2}=0$, so from Eq. (22) $\widetilde{\delta}=0$ or $\pi$ which implies the clock angle $\delta^{*}(0)=0$ or $\pi$.

(3) Minimize $a_{T}$ given in Eq. (27) for a hybrid sail of mass $m_{0}$ and determine the optimum sail pitch angle $\alpha^{*}(0)$ and keep it fixed for mission lifetime $\tau_{m}$ i.e., $\alpha^{*}(t)=\alpha^{*}(0)$ for $0 \leq t \leq \tau_{m}$. The normal to the hybrid sail $\mathbf{n}$, and unit vector $\mathbf{m}$ along the SRP force given in Eq. (20) become

$$
\begin{aligned}
& \mathbf{n}^{b}=\left[\begin{array}{lll}
\cos \alpha^{*}(0) & 0 & \pm \sin \alpha^{*}(0)
\end{array}\right]^{T} \\
& \mathbf{m}^{b}=\left[\begin{array}{lll}
\cos \theta^{*}(0) & 0 & \pm \sin \theta^{*}(0)
\end{array}\right]^{T}
\end{aligned}
$$

where $\theta^{*}(0)$ is calculated using Eq. (9).

(4) Calculate the SRP acceleration $a_{s}$ and SEP acceleration $a_{T}$ for a hybrid sail of mass $m$ from Eqs. (7) and (23) respectively at the optimum sail pitch angle and clock angle $\delta^{*}(t)=\widetilde{\delta}=0(\pi)$.

(5) Calculate the consumed propellant mass $m_{\text {prop }}(t)$ up to time $t$. 


$$
m_{\text {prop }}(t)=m_{0}\left(1-\exp \left(-\frac{1}{I_{s p} g_{0}} \int_{0}^{t} \dot{a}_{T} d t\right)\right)
$$

so that the instantaneous mass of the hybrid sail $m=m_{0}-m_{\text {prop }}$.

(6) Calculate electric thruster direction at time $t$

$$
\mathbf{u}^{b}=\left[\begin{array}{lll}
u_{1} & 0 & u_{3}
\end{array}\right]^{T}=\frac{1}{a_{T}}\left[\mathbf{a}_{g c}^{b}-a_{s} \mathbf{m}^{b}\right]
$$

(7) $t=t+\triangle t$. If $t<\tau_{m}$ go to step 4; otherwise the calculation is over.

\section{Option 2}

In option 2, the optimum sail pitch angle $\alpha^{*}(t)$ at each instant $t$ is determined using the instantaneous mass $m$ during the mission lifetime, instead of fixing it at $\alpha^{*}(0)$. In this case both $\mathbf{n}$ and $\mathbf{u}$ will be varied to keep the hybrid sail at an artificial equilibrium point $\mathbf{r}_{0}$. The algorithm in this case works similarly to option 1 except step (3) and step (7) should be replaced accordingly:

(3) Minimize $a_{T}$ given in Eq. (23) with $\delta^{*}(t)=\widetilde{\delta}=0$ or $\pi$ and determine the optimum sail pitch angle $\alpha^{*}(t)$ using the instantaneous mass $m$ of the hybrid sail. The unit vectors $\mathbf{n}^{b}$ and so $\mathbf{m}^{b}$ in this case will vary with time as

$$
\begin{aligned}
& \mathbf{n}^{b}=\left[\begin{array}{lll}
\cos \alpha^{*}(t) & 0 & \pm \sin \alpha^{*}(t)
\end{array}\right]^{T} \\
& \mathbf{m}^{b}=\left[\begin{array}{lll}
\cos \theta^{*}(t) & 0 & \pm \sin \theta^{*}(t)
\end{array}\right]^{T}
\end{aligned}
$$

where $\theta^{*}(t)$ is calculated using $\alpha^{*}(t)$.

(7) $t=t+\triangle t$. If $t<\tau_{m}$ go to step 3 ; otherwise the calculation is over.

\section{Linear Stability Analysis for the Hybrid Sail}

\section{A. Linearized System}

To determine the local stability property of an AEP $\mathbf{r}_{0}$, the variational equations in the vicinity of an equilibrium point are derived. Such linearized variational equations are obtained by replacing the nonlinear system Eq. (15) by a linear system around the equilibrium point $\mathbf{r}_{0}$. Using the transformation $\mathbf{r}=\mathbf{r}_{0}+\delta \mathbf{r}$ for linearization (in the xz-plane) and assuming the attitude of the hybrid sail $\mathbf{n}^{a}$ and thruster pointing of the SEP system $\mathbf{u}^{a}$ are not perturbed, so as to restrict the stability analysis in the sense of Lyapunov, Eq. (15) can be rewritten in the form 


$$
\frac{d^{2} \delta \mathbf{r}}{d t^{2}}+2 \boldsymbol{\omega} \times \frac{d \delta \mathbf{r}}{d t}+\nabla U\left(\mathbf{r}_{0}+\delta \mathbf{r}\right)=\mathbf{a}_{S}\left(\mathbf{r}_{0}+\delta \mathbf{r}, \mathbf{n}^{a}\right)+\mathbf{a}_{S E P}\left(\mathbf{r}_{0}+\delta \mathbf{r}, \mathbf{u}^{a}\right)
$$

The gradient of the potential and the acceleration vectors due to SRP and SEP can be expanded in Taylor series about the equilibrium point $\mathbf{r}_{0}$ to a first order as

$$
\begin{aligned}
\nabla U\left(\mathbf{r}_{0}+\delta \mathbf{r}\right) & =\nabla U\left(\mathbf{r}_{0}\right)+\left[\frac{\partial \nabla U}{\partial \mathbf{r}}\right]_{\mathbf{r}_{0}} \delta \mathbf{r}+O\left(|\delta \mathbf{r}|^{2}\right) \\
\mathbf{a}_{S}\left(\mathbf{r}_{0}+\delta \mathbf{r}, \mathbf{n}^{a}\right) & =\mathbf{a}_{S}\left(\mathbf{r}_{0}\right)+\left[\frac{\partial \mathbf{a}_{S}(\mathbf{r})}{\partial \mathbf{r}}\right]_{\left(\mathbf{r}_{0}, \mathbf{n}^{a}\right)} \delta \mathbf{r}+O\left(|\delta \mathbf{r}|^{2}\right) \\
\mathbf{a}_{S E P}\left(\mathbf{r}_{0}+\delta \mathbf{r}, \mathbf{u}^{a}\right) & =\mathbf{a}_{S E P}\left(\mathbf{r}_{0}\right)+\left[\frac{\partial \mathbf{a}_{S E P}}{\partial \mathbf{r}}\right]_{\left(\mathbf{r}_{0}, \mathbf{u}^{a}\right)} \delta \mathbf{r}+O\left(|\delta \mathbf{r}|^{2}\right)
\end{aligned}
$$

Assuming the acceleration $\mathbf{a}_{S E P}$ is fixed with respect to the perturbation $\delta \mathbf{r}$, we have

$$
\left[\frac{\partial \mathbf{a}_{S E P}}{\partial \mathbf{r}}\right]_{\mathbf{r}_{0}}=0
$$

Substituting Eqs. (33) and (34) into Eq. (32) and using the artificial equilibrium condition of Eq. (16), then the linear variational equation around an AEP $\mathbf{r}_{0}$ is obtained as

$$
\frac{d^{2} \delta \mathbf{r}}{d t^{2}}+2 \boldsymbol{\omega} \times \frac{d \delta \mathbf{r}}{d t}-K \delta \mathbf{r}=0
$$

where

$$
K=-\left[\frac{\partial \nabla U}{\partial \mathbf{r}}\right]_{\mathbf{r}_{0}}+\left[\frac{\partial \mathbf{a}_{S}(\mathbf{r})}{\partial \mathbf{r}}\right]_{\left(\mathbf{r}_{0}, \mathbf{n}^{a}\right)}
$$

For an artificial equilibrium point $\mathbf{r}_{0}$ in the xz-plane, the explicit expression for $K$ is given in the Appendix.

By letting $\mathbf{X}=(\delta \mathbf{r}, \delta \dot{\mathbf{r}})^{T}$, the linear system is $\dot{\mathbf{X}}=A(t) \mathbf{X}$. The Jacobian matrix $A(t)$ in the neighborhood of $\mathbf{r}_{0}$ is given by

$$
A(t)=\left(\begin{array}{cc}
0 & I \\
K & \Omega
\end{array}\right), \quad \Omega=\left(\begin{array}{ccc}
0 & 2 & 0 \\
-2 & 0 & 0 \\
0 & 0 & 0
\end{array}\right)
$$

The Jacobian matrix is constant when the dynamics of the pure sail are linearized in the CRTBP. However, the linearizaion for a hybrid sail in the CRTBP is a time varying system as the matrix $K$ given in Eq. (36) contains mass (time) varying parameters. The necessary condition for asymptotically stability of a linear time varying system is that for any $t>t_{0}{ }^{17}$

$$
\int_{t_{0}}^{t} \operatorname{tr} A(\tau) d \tau \rightarrow-\infty \quad \text { as } \quad t \rightarrow \infty
$$

\section{4 of 27}


where $\operatorname{tr} A(\tau)$ is the trace of $A(t)$. Since for the hybrid sail

$$
\int_{t_{0}}^{t} \operatorname{tr} A(\tau) d \tau=0
$$

it can be concluded that the linear time-varying system of the hybrid sail is not asymptotically stable, as is expected since there is no natural dissipation. One approach to investigate the instability of slowly time-varying linear systems is to employ a freezing-time method. ${ }^{18}$ In this approach, the time varying parameters (e.g., mass $m$ for a hybrid system) are fixed at their current values during each instant of time $t=t_{0}, t_{1}, t_{2}, \cdots, \tau_{m}$ and the Jacobian matrix $A\left(t_{i}\right)$ will be treated as constant for each interval $t_{i}$ to $t_{i+1}$. Then, the eigenvalues of the constant matrix $A\left(t_{i}\right)$ resulting from its characteristic equation are examined for instability. The instability properties of the time-varying system are the same as those of the frozen-time system provided that the eigenvalues of $A(t)$ are bounded away from the imaginary axis for all $t \geq 0$ (i.e., eigenvalues do not cross the imaginary axis) and if $\sup _{t \geq 0}\|\dot{A}(t)\|$ (i.e., the norm of the time derivative of matrix $\mathrm{A}(\mathrm{t}))$ is sufficiently small. ${ }^{18}$

\section{B. Stability Analysis at $t_{0}=0$}

To determine the stability of the linear system $\dot{\mathbf{X}}=A\left(t_{0}\right) \mathbf{X}$, the Jacobian matrix $A(t)$ time dependance is frozen at $t_{0}=0$ by substituting $m=m_{0}$ in the matrix $K$ of Eq. (36), so that $K$ may be written as

$$
K\left(t_{0}\right)=\left(\begin{array}{ccc}
k_{1} & 0 & k_{3} \\
0 & k_{5} & 0 \\
k_{7} & 0 & k_{9}
\end{array}\right)
$$

The characteristic equation of $A\left(t_{0}\right)$ in $\lambda$ is given by

$$
\left|A\left(t_{0}\right)-\lambda I_{6}\right|=\lambda^{6}+p_{2} \lambda^{4}+p_{1} \lambda^{2}+p_{0}=0
$$

where

$$
\begin{aligned}
& p_{2}=4-k_{1}-k_{5}-k_{9} \\
& p_{1}=k_{1} k_{5}-k_{3} k_{7}-4 k_{9}+k_{1} k_{9}+k_{5} k_{9} \\
& p_{0}=k_{3} k_{5} k_{7}-k_{1} k_{5} k_{9}
\end{aligned}
$$

If we define $\xi=\lambda^{2}$, then the characteristic equation becomes cubic in $\xi$ such that

$$
\xi^{3}+p_{2} \xi^{2}+p_{1} \xi+p_{0}=0
$$

The discriminant of the cubic Eq. (45) can be then defined as 


$$
D=4 p_{0} p_{2}^{3}-p_{1}^{2} p_{2}^{2}+4 p_{1}^{3}-18 p_{0} p_{1} p_{2}+27 p_{0}^{2}
$$

The roots of the cubic Eq. (45) in $\xi$ are real if the discriminant $D \leq 0$, or alternatively one real root and a pair of complex conjugate roots if $D>0$. However, if all the roots of the cubic equation are real i.e., $D \leq 0$, then by Descartes' rule of signs, ${ }^{19}$ the number of positive real roots (including multiplicity) is equal to the number of sign changes of the sequence $p_{0}, p_{1}, p_{2}$ in Eq. (45).

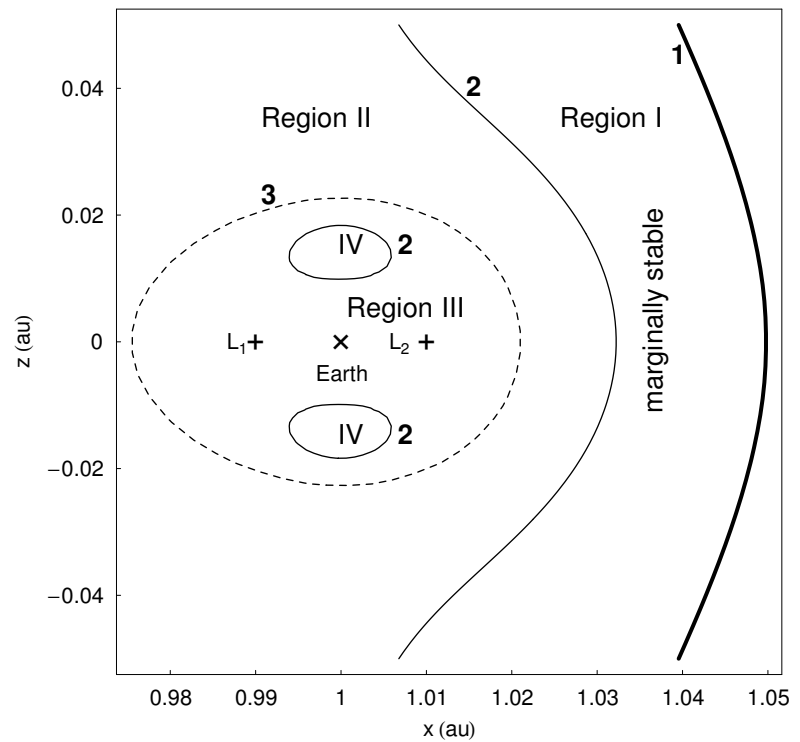

Figure 7. Regions I-IV in the Sun-Earth three-body system are classified according to the stability of artificial equilibria for a hybrid sail. 1,2 and 3 represent contours of $D=0, p_{0}=0$ and $p_{1}=0$ respectively.

The nature of the artificial equilibria (in the xz-plane) for the Sun-Earth CRTBP, where the third body is a hybrid sail is shown in Fig. 7. We label the regions as:

(a) Region I-if the discriminant $D<0$ and $p_{0}>0, p_{1}>0, p_{2}>0$, then by Descartes' rule of signs with no sign change of the coefficient sequence of the cubic equation, all the roots of Eq. (45) are negative. Therefore, the spectrum of the Jacobian $A\left(t_{0}\right)$ is centres given by

$$
\left\{ \pm i \lambda_{1}, \pm i \lambda_{2}, \pm i \lambda_{3}\right\} \quad \text { Region I marginally stable }
$$

(b) Region II-if the discriminant $D<0$ and $p_{0}<0, p_{1}>0, p_{2}>0$ or in Region III-if the discriminant $D<0$ and $p_{0}<0, p_{1}<0, p_{2}>0$, then by Descartes' rule of signs with one sign change of the coefficient sequence of the cubic equation, the spectrum of the Jacobian is centeres crossed with saddles

$$
\left\{ \pm i \lambda_{1}, \pm i \lambda_{2}, \pm \lambda_{r_{1}}\right\} \quad \text { Region II and III unstable }
$$


(c) Region IV-if the discriminant $D<0$ and $p_{0}>0, p_{1}<0, p_{2}>0$, then by Descartes' rule of signs with two sign changes of the coefficient sequence of the cubic Eq. (45), here the spectrum is

$$
\left\{ \pm i \lambda_{1}, \pm \lambda_{r_{1}}, \pm \lambda_{r_{2}}\right\} \quad \text { Region IV unstable }
$$

The hybrid sail in Region I does not use SRP as the pitch angle $\alpha^{*}(0)$ becomes approximately/or equal to $90^{\circ}$ to minimize the thrust acceleration $a_{T}$ from the SEP system. Therefore, in this region the hybrid sail acts as a pure SEP system and the marginally stable region (centers) of the pure SEP system are recovered. ${ }^{12}$

During the mission life at an artificial equilibria location $\mathbf{r}_{0}$, the mass $m$ of the hybrid sail changes according to Eq.(29). Hence the matrix $A(t)$, and its eigenvalues, also change with $m$. Simulations run for different artificial equilibria in Regions II-IV with corresponding mass variation show very slow variation and no sign change in the roots of the cubic Eq.(45) during the mission life. Thus, no eigenvalues cross the imaginary axis and the small parameter variations in $A(t)$ during the mission life implies instability of Regions II-IV.

\section{Evaluation of Hybrid Sail Performance}

This section compares the sizing of a hybrid sail, pure SEP system and pure sail system for a polar (Earth) observer mission. In the polar observer mission, AEPs along the polar axis, high above the $L_{1}$ side of the Earth are selected in the Sun-Earth system. Such equilibrium locations have been proposed by McInnes and others ${ }^{8,20}$ in the case of a pure sail for continual, low resolution imaging of high latitude regions of Earth and for polar telecommunication services at $L_{2} \cdot{ }^{10}$

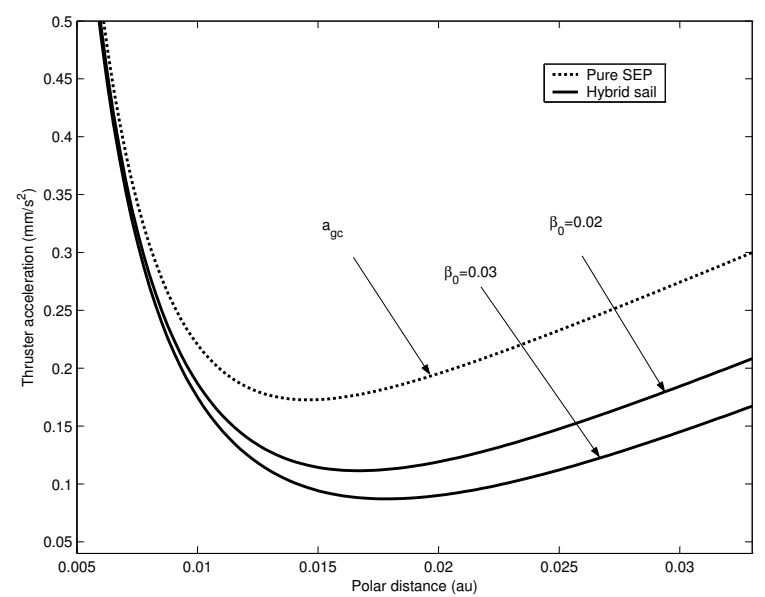

Figure 8. Thrust acceleration magnitude required from pure SEP and hybrid sail (at $t=0$ ) at artificial equilibrium points above $L_{1}$ along the Earth's polar axis (north pole at summer solstice).

The magnitude of the required acceleration $a_{g c}$ for an AEP along the polar axis is shown by the dotted lines in Fig. 8. It can be seen that $a_{g c}$ has a minimum value along the polar axis due to the Sun-Earth three body dynamics. To generate an AEP the pure SEP system alone provides the acceleration $a_{g c}$. For the hybrid 
sail, the thruster system provides less acceleration $a_{T}\left(\alpha^{*}(0)\right)$ shown by the solid lines in Fig. 8 since part of the acceleration is provided by the SRP to achieve $a_{g c}$.

The initial (wet) mass for each propulsion system is calculated to position a spacecraft on an AEP with a fixed payload mass $m_{p l}$ and fixed mission life $\tau_{m}$. The total payload mass (including a small optical imager and spacecraft bus) of $100 \mathrm{~kg}$ is assumed for the polar observer mission. The pure sail is a propellantless system, unlike pure SEP and the hybrid sail, and the pure sail mission life is limited only by the sail film. Hence, for the pure sail system only the payload mass $m_{p l}$ is fixed. Some near-term pure sail missions are envisaged with a sail lifetime of at least 5 years. $^{21}$

\section{A. Pure Sail}

For a pure sail, the total mass $m_{0}$ can be decomposed into the sail assembly mass $m_{S}$ (sail film, booms and deployment module) and the payload mass $m_{p l}$. The sail assembly mass $m_{S}$ is usually written in terms of sail area and sail assembly loading $\sigma_{S}$ (defined as mass per unit area of the sail assembly), a key technology parameter that is a measure of the sail film thickness and lightness of the booms and deployment module. For a fixed $m_{p l}$ and $\sigma_{S}$ as given parameters, the initial mass can be calculated for a given equilibrium location $\mathbf{r}_{0}$ as

$$
m_{0}=m_{p l}+m_{S}=m_{p l}+\sigma_{S}\left[\frac{m_{p l}}{\sigma_{T}-\sigma_{S}}\right]
$$

where $\sigma_{T}=\frac{m_{0}}{A_{S}}$ is the total (pure) sail loading. It can be calculated from the appropriate sail lightness number which is uniquely determined by the chosen equilibria location $\mathbf{r}_{0}$ and the sail film reflectivity $\tilde{r}_{S} \cdot{ }^{22}$

\section{B. Pure SEP}

The polar observer mission is a long term and large $\Delta V$ mission. To reduce $m_{0}$ for a given $m_{p l}$, in the case of pure SEP and the hybrid sail, electric thruster selection should be made to reduce the propellant mass $m_{\text {prop }}$ and the electric propulsion inert mass. Ion thrusters, among various kinds of electric propulsion systems, are well-suited because of their potential for providing high $I_{s p} \approx 3200 \mathrm{~s}$ (reduces the propellant requirement), high efficiency and higher total impulse. ${ }^{23}$ Higher efficiency for a given $I_{s p}$ and thrust level reduces the input power of the SEP system, while higher total impulse reduces the number of thrusters, and thus also the inert mass of the SEP system. For a pure SEP system with TFSC technology as a power source, the initial mass $m_{0}$ breakdown can be written as

$$
m_{0}=m_{p l}+m_{\text {Tank }}+n_{T h} \cdot m_{\text {inert }}+m_{\text {prop }}+m_{T F}
$$

where $m_{\text {Tank }}$ is the (empty) propellant tank mass, $m_{\text {prop }}$ the propellant mass, $m_{T F}$ the TFSC mass, and $m_{\text {inert }}$ the inert mass of SEP system including the mass of the thruster, power processor unit (PPU), thermal 
system for the PPU, Digital Control and Interface unit (DCIU) and cabling/propellant feeding system. $n_{T h}$ is the number of thrusters. Two thrusters are assumed in series, each with an operating life of 2.5 years. In order to maintain the artificial equilibria $\mathbf{r}_{0}$, the constant acceleration $a_{g c}\left(\mathbf{r}_{0}\right)$ should be provided by the pure SEP system during mission life $\tau_{m}$, and thus the propellant mass consumed $m_{\text {prop }}$ is given by

$$
m_{\text {prop }}=m_{0}\left(1-\exp \left(\frac{-\dot{a}_{g c} \tau_{m}}{I_{s p} g_{0}}\right)\right)
$$

In the case of ion thrusters the approximate relations in Eq.(48) are given by

$$
\begin{aligned}
m_{\text {Tank }} & =0.1 m_{\text {prop }} \\
m_{\text {inert }} & =k_{e} P_{e, \max } \\
m_{T F} & =\sigma_{T F} A_{T F}
\end{aligned}
$$

The reasonable assumption is made that the mass of the propellant tank $m_{\text {Tank }}$ is $10 \%$ of the propellant mass. ${ }^{24}$ The specific mass $k_{e}$ is assumed to be $20 \mathrm{~kg} / \mathrm{kW}$ (as for the NSTAR class engine ${ }^{25}$ ). The areal density $\sigma_{T F}$ of the TFSC is assumed to be $100 \mathrm{~g} / \mathrm{m}^{2}{ }^{14}$ The thin film solar cell area $A_{T F}$ is selected by using the maximum power level required $P_{e, \max }$ (or maximum thrust $T_{\max }$ ), $W$ the solar flux at the AEP $\mathbf{r}_{0}$, and efficiency $\eta_{T F}$ (i.e., converting solar energy into electrical energy) as follows

$$
A_{T F}=\frac{P_{e, \max }}{W \eta_{T F}}
$$

In Eq. (51) the TFSC area is assumed to be pointed at the Sun while the ion thruster is firing in the desired direction to maintain the artificial equilibrium $\mathbf{r}_{0}$. Although TFSC technology gives larger $A_{T F}$ as compared to wafer based technology for a required $P_{e, \max }$, due to its low efficiency $\eta_{T F}=0.05$, it results in a lower mass $m_{T F}$ due to the small value of $\sigma_{T F}$. If $\eta_{e}=0.7^{26}$ is the efficiency of converting electrical energy into constant exhaust velocity $v_{e}=I_{s p} g_{0}$, then

$$
P_{e, \max }=\frac{T_{\max } v_{e}}{2 \eta_{e}}=\frac{m_{0} \dot{a}_{g c} I_{s p} g_{0}}{2 \eta_{e}}
$$

The initial (wet) mass $m_{0}$ for a pure SEP system then can be written in terms of $m_{p l}$ and $\tau_{m}$ by substituting Eqs. (49-52) into Eq. (48) to obtain

$$
m_{0}=\frac{m_{p l}}{1-1.1\left(1-\exp \left(\frac{-a_{g c} \tau_{m}}{I_{s p} g_{0}}\right)\right)-\frac{a_{g c} I_{s p} g_{0}}{2 \eta_{e}}\left(k_{e} n_{T h}+\frac{\sigma_{T F}}{W \eta_{T F}}\right)}
$$

\section{Hybrid Sail}

The initial mass breakdown for a hybrid sail is assumed as 


$$
m_{0}=m_{p l}+m_{\text {Tank }}+n_{T h}\left(m_{\text {inert }}+m_{\text {gimbal }}\right)+m_{\text {prop }}+m_{T F}+m_{S}
$$

Here the gimbal mass $m_{\text {gimbal }}$ for each engine is assumed to be $30 \%$ of inert mass of the thruster system $m_{\text {inert }} .{ }^{24} \mathrm{~A}$ gimbal is required to actuate the thruster relative to the sail assembly to maintain equilibrium. With a given $m_{0}$, the propellant mass $m_{\text {prop }}$ required to maintain the artificial equilibrium for a mission life $\tau_{m}$ can be calculated using the algorithms described in section III.B. In Eq. (54) for $m_{\text {Tank }}, m_{\text {inert }}$ and $m_{T F}$, the same approximate relations for the pure SEP system given in Eq. (50) are assumed. However, with a given $m_{0}$, we replace the maximum thrust level by $T_{\max }=m_{0} a_{T}\left(\alpha^{*}(0)\right)$ in Eq. (52) and moreover divide Eq. (51) by $\cos \left(\alpha^{*}(0)\right)$, to calculate $m_{\text {inert }}$ and $m_{T F}$ respectively since the TFSC is attached to the sail and so is not Sun-pointing. Also with a given $m_{0}$, the sail mass $m_{S}$ in Eq.(54) is given by

$$
m_{S}=\sigma_{S} A_{T}=\sigma_{S}\left(\frac{m_{0} \beta_{0}}{\sigma^{*}}\right)
$$

In summary, for a given $\beta_{0}, \tau_{m}, \sigma_{S}$ and an initial guess $m_{0}$, the payload mass $m_{p l}$ can be calculated using Eq. (54). A simple shooting method is used to determine $m_{0}$ for different artificial equilibria along the polar axis so that the payload mass $m_{p l}$ becomes $100 \mathrm{~kg}$.

Figure 9 shows that the minimum initial mass $m_{0}$ along the polar axis for a pure SEP it is located at $0.0145 \mathrm{AU}$, for a pure sail with sail film reflectivity $\tilde{r}_{S}=0.9$ it is located at $0.025 \mathrm{AU}$ and for a hybrid sail with $\beta_{0}=0.03$ it is located at 0.0183 AU. For the pure SEP system, the minimum $m_{0}$ at 0.0145 AU is due to the minimum $a_{g c}$ at $0.0145 \mathrm{AU}$, see Fig. 8. For a pure sail, the minimum is shifted to 0.025 AU due to the variation of SRP acceleration with $\alpha$. For a hybrid sail, the location for minimum $m_{0}$ depends upon the minimum location of $a_{T}\left(\alpha^{*}(0)\right)$, see Figs. 8 and 9 . Figure $9(\mathrm{a}-\mathrm{c})$ also shows the dependance of $m_{0}$ for the pure sail and hybrid sail systems with variation of the sail assembly loading. The vertical line shows that the pure sail cannot be placed along polar axis below $0.015 \mathrm{AU}$. The sail assembly loading of $15 \mathrm{~g} / \mathrm{m}^{2}$, $10 \mathrm{~g} / \mathrm{m}^{2}$ and $5 \mathrm{~g} / \mathrm{m}^{2}$ may be assumed for near, mid term and far term sails. ${ }^{24}$ Figure 9 shows that for a near term sail assembly loading of $13.75 \mathrm{~g} / \mathrm{m}^{2}$, the minimum initial mass $m_{0}$ for a pure SEP (with maximum power level of $1.5 \mathrm{~kW}$ ) and a pure sail (with sail length of $170 \mathrm{~m}$ ) becomes equal to $500 \mathrm{~kg}$, and the hybrid sail clearly has a lower initial mass of $365 \mathrm{~kg}$ (sail length of $85 \mathrm{~m}$ and maximum power level of $715 \mathrm{~W}$ ) at an optimum distance of $0.0183 \mathrm{AU}$, and thus a lower launch mass and higher resolution for imaging than the pure sail. Figure $9 \mathrm{c}$ shows that for a far term sail assembly loading i.e., $5 \mathrm{~g} / \mathrm{m}^{2}$, the hybrid sail has a significant improvement in payload fraction below $0.015 \mathrm{AU}$ along the polar axis as compared to the pure SEP, and also a higher resolution for imaging than a pure sail.

Table 1 shows the initial mass breakdown with a mid term sail assembly loading, for station keeping a $100 \mathrm{~kg}$ payload mass at a polar distance of $0.01831 \mathrm{AU}$ (see Fig. 9b). The hybrid sail total initial mass $m_{0}$ at this AEP is less than that of the pure SEP and pure sail systems. The hybrid sail total mass is less than 


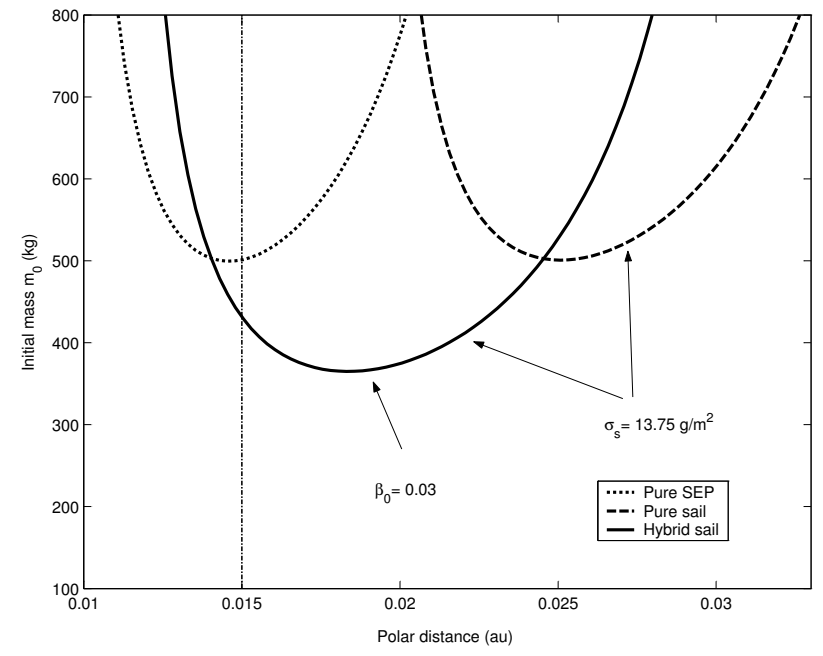

(a)

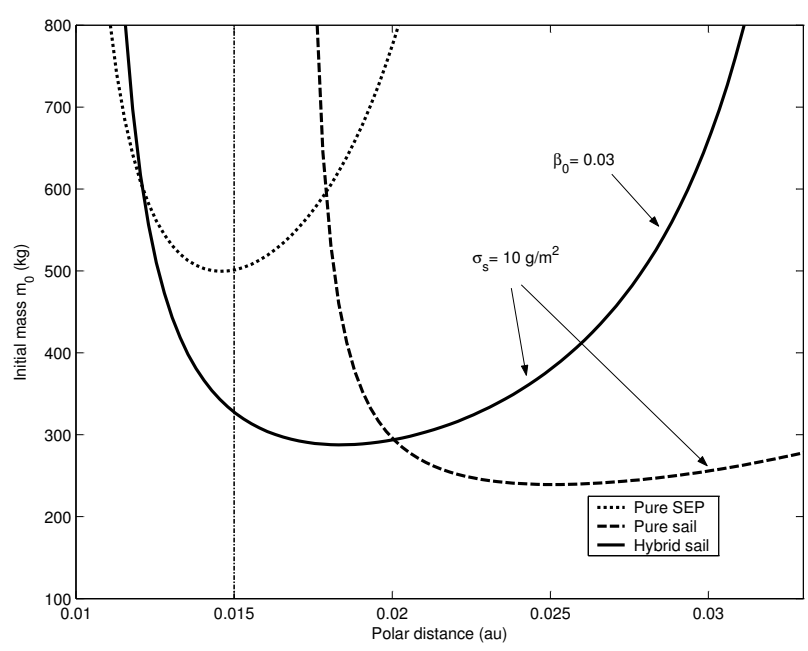

(b)

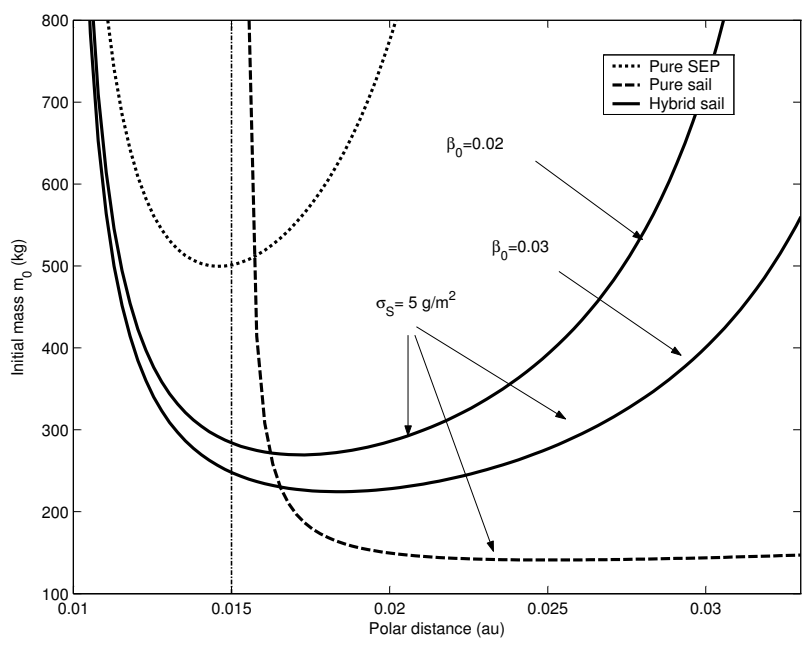

(c)

Figure 9. Spacecraft initial (wet) mass required for an AEP for a $100 \mathrm{~kg}$ payload for a polar observer mission (above $L_{1}$ ). Five year mission lifetime is considered for a pure SEP and hybrid sail. Initial mass variation for a pure and hybrid sail with sail assembly loading equal to a) $13.75 \mathrm{~g} / \mathbf{m}^{2}$ b) $10 \mathrm{~g} / \mathbf{m}^{2}$ and c) $5 \mathrm{~g} / \mathbf{m}^{2}$ are also shown. 
Table 1. Mass breakdown for three different propulsion systems for a spacecraft stationed at a polar distance 0.01831 AU along the polar axis above $L_{1}$. A fixed payload mass and fixed mission life of 5 years is assumed in the case of pure SEP and hybrid sail.

\begin{tabular}{lccc}
\hline \hline Subsystem & $\begin{array}{c}\text { Pure sail }^{\mathrm{a}} \\
\text { mass budget, kg }\end{array}$ & $\begin{array}{c}\text { Pure SEP } \\
\text { mass budget, kg }\end{array}$ & Hybrid sail $^{\mathrm{a}}$ \\
\hline$m_{p l}$ & 100 & 100 & 100 \\
$m_{\text {prop }}$ & & 376 & 92 \\
$m_{\text {inert }}($ 1st thruster $)$ & $51.5^{b}$ & $11.3^{b}$ \\
$m_{\text {gimbal }}$ & & 3.4 \\
$m_{\text {Tank }}$ & & 37.5 & 9.2 \\
$m_{\text {TF }}$ & & 4.5 & 1.2 \\
$m_{\text {inert }}(2$ nd thruster $)$ & 360 & 51.5 & $14.7^{c}$ \\
$m_{S}$ & 460 & & 56.5 \\
$m_{0}$ & & 621 & 288 \\
(Total initial mass $)$ & & & \\
\hline \hline
\end{tabular}

${ }^{\mathrm{a}}$ Pure sail length $=190 \mathrm{~m}$ and hybrid sail length $=75 \mathrm{~m}$; sail assembly loading $\sigma_{S}=10 \mathrm{~g} / \mathrm{m}^{2}$.

${ }^{b} P_{e, \max }=564 \mathrm{~W}$ for hybrid sail, $P_{e, \max }=2.58 \mathrm{~kW}$ for pure SEP

${ }^{c}$ Includes also gimbal mass 
the total mass of the pure SEP as the saving in propellant mass and inert mass of the thruster system for the hybrid sail, totaling $389 \mathrm{~kg}$, is greater than the additional penalty of the sail assembly mass $m_{S}=56.5 \mathrm{~kg}$ compared to pure SEP. The smaller inert mass of the thruster system for the hybrid sail is due to the lower maximum power required as compared to the maximum power for a pure SEP thruster system. The hybrid sail total mass is less than the total mass of the pure sail as the saving from the sail mass for the hybrid sail, by reducing the sail length, is greater than the penalty of propellant and inert mass of the thruster system needed for the hybrid sail. Table 1 shows that the mass of TFSC area is small compared to the inert mass, tank and propellant of SEP system. ${ }^{14}$

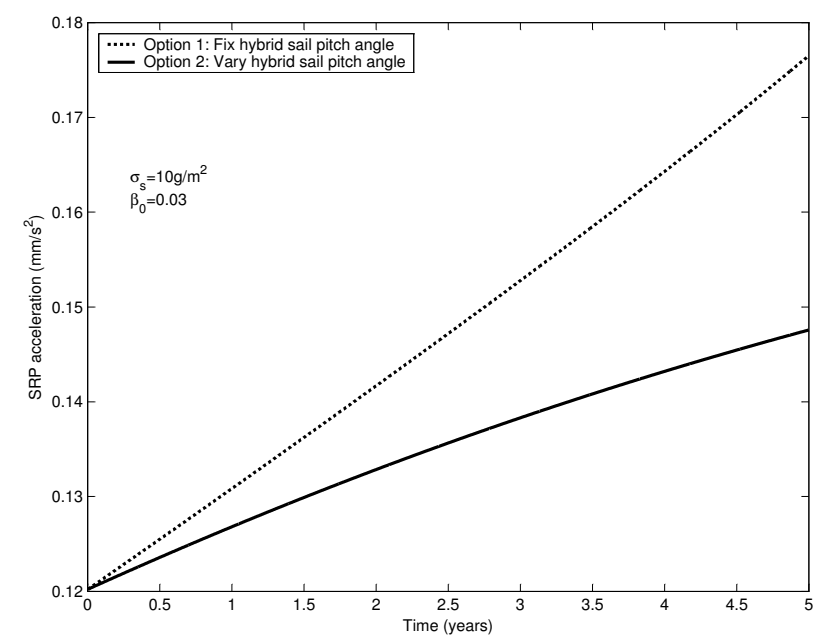

(a)

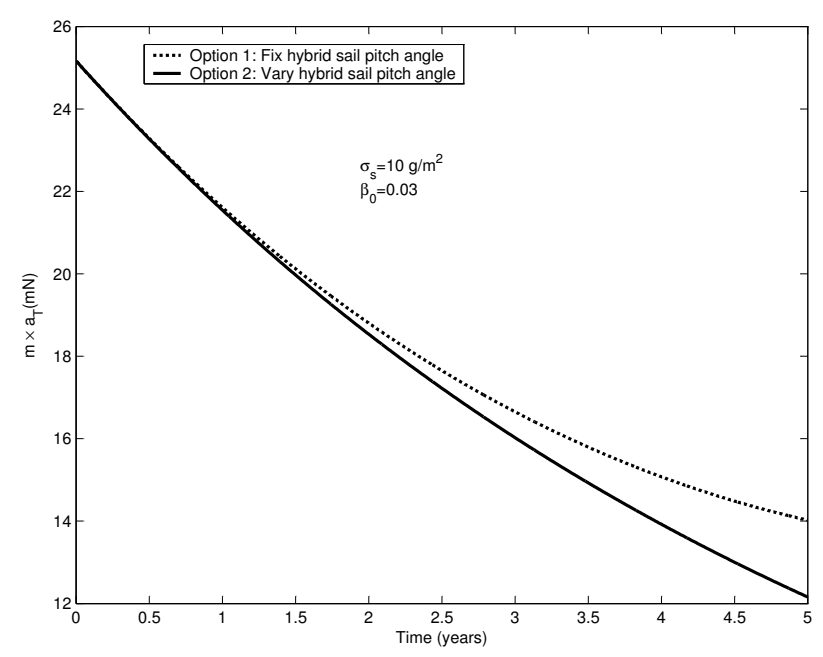

(c)

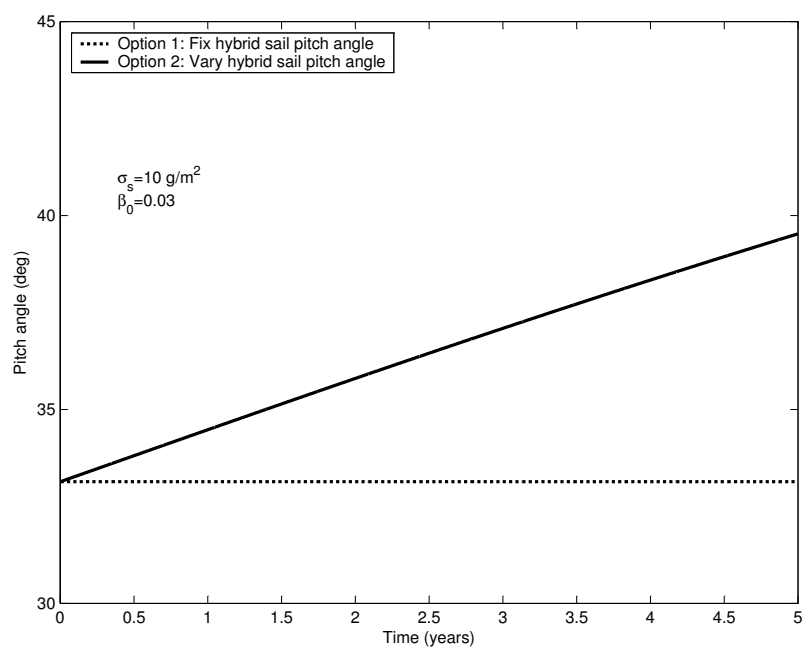

(b)

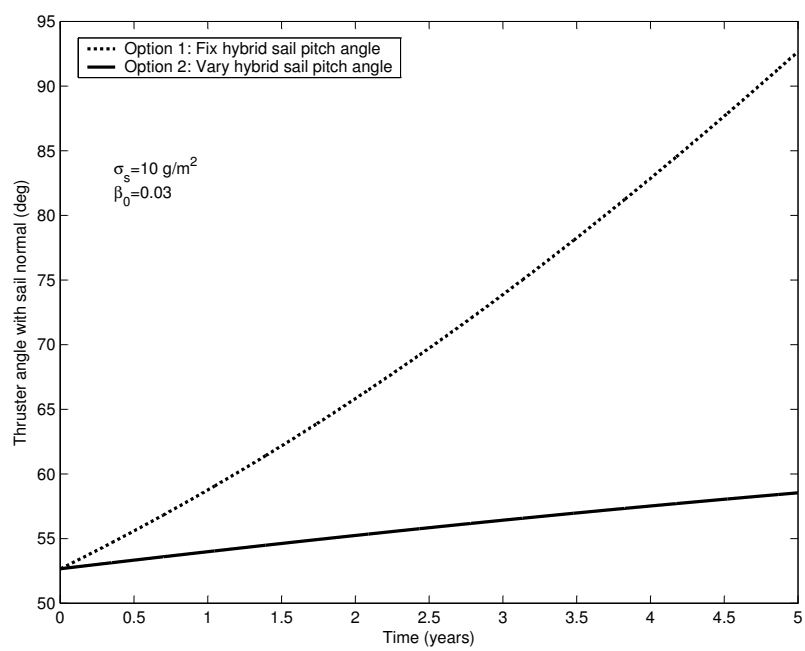

(d)

Figure 10. Hybrid sail a) SRP acceleration magnitude b) Pitch angle c) ion thruster force d) ion thruster firing angle w.r.t. sail normal, during mission life stationed at polar distance $0.01831 \mathrm{AU}$ along the polar axis above $L_{1}$.

Figure 10 shows the hybrid sail parameter variation during the mission life when it is in a static equilibrium 
with either of the two equilibria strategies (option 1 and option 2). Figure 10a shows the increase of SRP magnitude $a_{s}$ due to the decrease of mass $m$ during the mission life. For option 2, there is a slow variation in $a_{s}$ due to the increase of the hybrid sail pitch angle $\alpha^{*}(t)$ as compared to its fixed pitch angle $\alpha^{*}(0)$ for option 1 (see Fig. 9b). Figures 10c and 10d show the required force and orientation of the SEP thruster system to maintain the equilibrium condition. Although the same orientation, 52.66 deg for this AEP, is required at the start of the mission in both options, there is less variation in the orientation of the ion thruster system relative to the sail normal for option 2. Option 2 is better than option 1 as the thruster plume does not interact with the sail film during the whole mission life (see Fig. 10d). Moreover, in option 2 less thrust is required during the whole mission life (see Fig. 10c) with fixed $I_{s p}$, which suggests less total propellant mass consumption, and hence less total initial mass $m_{0}$. However, for both options, the ion thruster system must have the capability of throttling down and a gimbal system is required to maintain equilibrium.

\section{Conclusion}

In this paper a new concept to generate artificial equilibrium points by using a hybrid solar sail in the circular restricted three-body problem has been analyzed. The key idea is that the required acceleration vector to keep the hybrid sail at an artificial equilibrium point is achieved by the vector sum of the solar radiation pressure acceleration and the solar electric propulsion acceleration vectors. We cast the problem to minimize the acceleration from the solar electric propulsion system (SEP) of the hybrid sail for a given sail lightness number. It is shown that the hybrid sail clock angle should be aligned with the clock angle of the required acceleration vector in order to minimize acceleration from the SEP system. Finally, the minimization problem for equilibrium reduces to numerically determining the optimum hybrid sail pitch angle. A linear stability analysis shows that the artificial equilibrium points for the hybrid sail are unstable in general, apart from some region where the equilibria are marginally stable. Moreover, the time varying parameter (mass variation) of the hybrid sail does not change the stability properties of the equilibria. It has been shown that the hybrid sail has a potential application of hovering above the $L_{1}$ point for real-time, low resolution images of the poles. The hybrid sail along the polar axis is found to have a lower sail length compared to a pure sail and a lower maximum power level as compared to a pure SEP system. For a near term sail assembly loading $\left(13.75 \mathrm{~g} / \mathrm{m}^{2}\right)$, the hybrid sail for the polar observer mission clearly demonstrates a greater payload mass fraction. Furthermore, the hybrid sail can be used to obtain higher resolution images by hovering in a region which is inaccessible for the pure sail.

\section{Appendix: Matrix K}

For the matrix $\frac{\partial \nabla U}{\partial \mathbf{r}}$ in Eq. (36) the terms are 


$$
\frac{\partial \nabla U}{\partial \mathbf{r}}=\left(\begin{array}{ccc}
U_{x x} & U_{x y} & U_{x z} \\
U_{y x} & U_{y y} & U_{y z} \\
U_{z x} & U_{z y} & U_{z z}
\end{array}\right)
$$

The acceleration due to solar pressure for a hybrid sail can be rewritten using Eqs. (12) and (7) as

$$
\mathbf{a}_{S}=\frac{1}{2} \beta_{0} \frac{m_{0}}{m} \frac{1-\mu}{r_{1}^{4}} \psi \mathbf{m}^{a}
$$

where

$$
\psi=\left[\left(g^{2}-h^{2}\right)\left(\mathbf{r}_{1} \cdot \mathbf{n}\right)^{4}+h^{2} r_{1}^{2}\left(\mathbf{r}_{1} \cdot \mathbf{n}\right)^{2}\right]^{1 / 2}
$$

The partial derivatives of Eq. (57) can be obtained as

$$
\frac{\partial \mathbf{a}_{S}}{\partial \mathbf{r}}=-2 \beta_{0} \frac{m_{0}}{m} \frac{(1-\mu)}{r_{1}^{5}} \psi\left(\begin{array}{lll}
\frac{(x+\mu)}{r_{1}} m_{x} & \frac{y}{r_{1}} m_{x} & \frac{z}{r_{1}} m_{x} \\
\frac{(x+\mu)}{r_{1}} m_{y} & \frac{y}{r_{1}} m_{y} & \frac{z}{r_{1}} m_{y} \\
\frac{(x+\mu)}{r_{1}} m_{z} & \frac{y}{r_{1}} m_{z} & \frac{z}{r_{1}} m_{z}
\end{array}\right)+\frac{1}{2} \beta_{0} \frac{m_{0}}{m} \frac{(1-\mu)}{r_{1}^{4}}\left(\begin{array}{lll}
\frac{\partial \psi}{\partial x} m_{x} & \frac{\partial \psi}{\partial y} m_{x} & \frac{\partial \psi}{\partial z} m_{x} \\
\frac{\partial \psi}{\partial x} m_{y} & \frac{\partial \psi}{\partial y} m_{y} & \frac{\partial \psi}{\partial z} m_{y} \\
\frac{\partial \psi}{\partial x} m_{z} & \frac{\partial \psi}{\partial y} m_{z} & \frac{\partial \psi}{\partial z} m_{z}
\end{array}\right)
$$

where

$$
\left(\begin{array}{c}
\frac{\partial \psi}{\partial x} \\
\frac{\partial \psi}{\partial y} \\
\frac{\partial \psi}{\partial z}
\end{array}\right)=\frac{2\left(g^{2}-h^{2}\right)\left(\mathbf{r}_{1} \cdot \mathbf{n}\right)^{3}+h^{2} r_{1}^{2}\left(\mathbf{r}_{1} \cdot \mathbf{n}\right)}{\psi}\left(\begin{array}{c}
n_{x} \\
n_{y} \\
n_{z}
\end{array}\right)+\frac{h^{2}\left(\mathbf{r}_{1} \cdot \mathbf{n}\right)^{2}}{\psi}\left(\begin{array}{r}
x+\mu \\
y \\
z
\end{array}\right)
$$

and $\mathbf{m}^{a}=\left[m_{x} m_{y} m_{z}\right]^{T}$ and $\mathbf{n}^{a}=\left[n_{x} n_{y} n_{z}\right]^{T}$ may be calculated as

$$
\begin{aligned}
\mathbf{m}^{a} & =\mathbf{C}_{b / a}^{T} \mathbf{m}^{b} \\
\mathbf{n}^{a} & =\mathbf{C}_{b / a}^{T} \mathbf{n}^{b}
\end{aligned}
$$

For an artificial equilibrium point $\mathbf{r}_{0}$ in the xz-plane, $\mathbf{m}^{b}$ and $\mathbf{n}^{b}$ are given by Eqs. (28) and (31) for option 1 and option 2 respectively. Furthermore, for $\mathbf{r}_{0}$ in xz-plane $y=n_{y}=m_{y}=0$, so the two matrices given in Eqs. (56) and (58) finally reduce to

$$
\left[\frac{\partial \nabla U}{\partial \mathbf{r}}\right]_{\mathbf{r}_{0}}=\left(\begin{array}{ccc}
U_{x x} & 0 & U_{x z} \\
0 & U_{y y} & 0 \\
U_{z x} & 0 & U_{z z}
\end{array}\right)
$$

and 


$$
\left[\frac{\partial \mathbf{a}_{S}}{\partial \mathbf{r}}\right]_{\left(\mathbf{r}_{0}, \mathbf{n}^{a}\right)}=-2 \beta_{0} \frac{m_{0}}{m} \frac{(1-\mu)}{r_{1}^{5}} \psi\left(\begin{array}{ccc}
\frac{(x+\mu)}{r_{1}} m_{x} & 0 & \frac{z}{r_{1}} m_{x} \\
0 & 0 & 0 \\
\frac{(x+\mu)}{r_{1}} m_{z} & 0 & \frac{z}{r_{1}} m_{z}
\end{array}\right)+\frac{1}{2} \beta_{0} \frac{m_{0}}{m} \frac{(1-\mu)}{r_{1}^{4}}\left(\begin{array}{ccc}
\frac{\partial \psi}{\partial x} m_{x} & 0 & \frac{\partial \psi}{\partial z} m_{x} \\
0 & 0 & 0 \\
\frac{\partial \psi}{\partial z} m_{z} & 0 & \frac{\partial \psi}{\partial z} m_{z}
\end{array}\right)
$$

so that $K$ in Eq. (36) can be calculated using Eqs. (60) and (61).

\section{Acknowledgments}

This work was funded by National Center for Physics, Quaid-i-Azam University, Islamabad, Pakistan. We acknowledgment useful discussions with Thomas J. Waters.

\section{References}

${ }^{1}$ McInnes, C., McDonald, A., Simmons, J., and McDonald, E., "Solar sail parking in restricted three-body systems," Journal of Guidance, Control and Dynamics, Vol. 17, No. 2, 1994, pp. 399-406.

${ }^{2}$ McInnes, C. R., "Artificial Lagrange points for a non-perfect solar sail," Journal of Guidance, Control and Dynamics, Vol. 22, No. 1, 1999, pp. 185-187.

${ }^{3}$ Morrow, E., Scheeres, D., and Lubin, D., "Solar sail orbit operations at asteroids," Journal of Spacecraft and Rockets, Vol. 38, No. 2, 2001, pp. 279-286.

${ }^{4}$ Baoyin, H. and McInnes, C., "Solar sail equilibria in the elliptical restricted three-body problem," Journal of Guidance, Control and Dynamics, Vol. 29, No. 3, 2006, pp. 538-543.

${ }^{5}$ Baoyin, H. and McInnes, C., "Solar sail halo orbits at the Sun-Earth artificial $\mathrm{L}_{1}$ point," Celestial Mechanics and Dynamical Astronomy, , No. 94, 2006, pp. 155-171.

${ }^{6}$ Waters, T. and McInnes, C., "Periodic orbits above the ecliptic in the solar sail restricted 3-body problem," Journal of Guidance, Control and Dynamics, Vol. 30, No. 3, 2007, pp. 687-693.

${ }^{7}$ Wan, C., "Solar Sail Geostorm Warning Mission Design," Proc. AAS/AIAA Spaceflight Mechanics Conference, Maui, Hawaii, No. AAS 04-107, Febraury, 2004.

${ }^{8}$ Crison, M. and Dittberner, G., "Advanced technologies for future environmental satellite systems," Paper 1.2, 13th Conference on Satelite Meterology and Oceanography, Norfolk, Virginia, 2004.

${ }^{9}$ McInnes, C. R., Solar sailing: technology, dynamics and mission applications, Springer Praxis, London, 1999, pp.250-256.

${ }^{10}$ Forward, R., "Statite: a spacecraft that does not orbit," Journal of Spacecraft and Rockets, Vol. 28, No. 5, 1991, pp. 606-611.

${ }^{11}$ Garbe, G. and Montgomery, E. E., "An Overview of NASA's Solar Sail Propulsion Project," 39th AIAA Joint Propulsion Conference and Exhibit, Huntsville, AL, , No. AIAA 2003-5274, July 20-23, 2004.

${ }^{12}$ Morimoto, M., Yamakawa, H., and Uesugi, K., "Artificial Equlibrium Points in the Low-Thrust Restricted Three-Body Problem," Journal of Guidance, Control and Dynamics, Vol. 30, No. 5, 2007, pp. 1563-1567.

${ }^{13}$ Morimoto, M., Yamakawa, H., and Uesugi, K., "Periodic orbits with Low-Thrust Propulsion in the Restricted Three-Body Problem," Journal of Guidance, Control and Dynamics, Vol. 29, No. 5, 2006, pp. 1131-1139.

${ }^{14}$ Leipold, M. and Götz, M., "Hybrid Photonic/Electric Propulsion," Kayser-Threde, GMBH, Rept. SOL4- TR-KTH-001, Munich, Germany, Jan. 2002. 
${ }^{15}$ Granata, J. E., Hausgen, P. E., Senft, D., Tlomak, P., and Merrill, J., "AFRL Thin Film Solar Cell Development and Upcoming Flight Experiments," Proc. 2005 IEEE Aerospace Conference, Big Sky, Montana, No. 1590, March 5-12 2005.

${ }^{16}$ Mengali, G. and Quarta, A., "Trajevtory Design with Hybrid Low-Thrust Propulsion System," Journal of Guidance, Control and Dynamics, Vol. 30, No. 2, March-April 2007, pp. 419-426.

${ }^{17} \mathrm{Wu}, \mathrm{M} .$, "On stability of linear time-varying systems," International Journal of Systems Science, Vol. 15, No. 2, 1984, pp. $127-150$.

${ }^{18}$ Skoog, R. and Lau, C., "Instability of Slowly Varying Systems," IEEE Transactions on Automatic Control, Vol. 17, No. 1, 1972, pp. 86-92.

${ }^{19}$ Råde, L. and Westergren, B., Mathematics Handbook for Science and Engineering, Studentlitteratur, Sweden, 3rd ed., 1995, pp. 64-65.

${ }^{20}$ McInnes, C., Eiden, M., Groepper, P., and Peacock, T., "Solar Sailing -Mission Opportunities and Innovative Technology Demonstartion," ESA Bulletin 108, 2001, pp. 58-65.

${ }^{21}$ Wie, B., Murphy, D., Paluszek, M., and Thomas, S., "Robust Attitude Control Systems Design for Solar Sails, Part 1: Propellantless Primary ACS," AIAA Guidance, Navigation, and Control Conference and Exhibit, Providence, Rhode Island, AIAA-2004-5010, Aug 16-19, 2004.

${ }^{22}$ Gurfil, P., Modern Astrodynamics, Elsevier Ltd, London, 1st ed., 2006, pp. 189-207.

${ }^{23}$ Frisbee, R., "Advanced Space Propulsion for the 21st Century," Journal of Propulsion and Power, Vol. 19, No. 6, 2003, pp. $1129-1154$.

${ }^{24}$ Gershman, R. and Seybold, C., "Propulsion Trades for Space Science Missions," Acta Astronautica, Vol. 45, No. 4-9, 1999 , pp. 541-548.

${ }^{25}$ Brophy, J., "Advanced Ion Propulsion Systems for Affordable Deep-Space Mission," Acta Astronautica, Vol. 52, No. 2-6, March 2003, pp. 309-316.

${ }^{26}$ S. Kitamura, Y. Ohkawa, Y. H. H. Y. and Miyazaki, K., "Overview and research status of the JAXA 150-mN ion engine," Acta Astronautica, Vol. 61, 2007, pp. 360-366. 